\title{
LRRK2 impairs PINK1/Parkin-dependent mitophagy via its kinase activity: pathologic insights into Parkinson's disease
}

Fiona Bonello, Sidi-Mohamed Hassoun, François Mouton-Liger, Yea Seul Shin, Adeline Muscat, Christelle Tesson, Suzanne Lesage, Philip M. Beart, Alexis Brice, Johannes Krupp, Jean-Christophe Corvol, Olga Corti*

${ }^{1}$ Institut du Cerveau et de la Moelle épinière, ICM, Paris, F-75013, France

${ }^{2}$ Inserm, U1127, Paris, F-75013, France

${ }^{3}$ CNRS, UMR 7225, Paris, F-75013, France

${ }^{4}$ Sorbonne Universités, Paris, F-75013, France

${ }^{5}$ Ipsen Innovation, Les Ulis, France

${ }^{6}$ AP-HP, Hôpital de la Pitié-Salpêtrière, Clinical Investigation Center for Neurology (CIC1422), Department of Neurology, Hôpital Pitié-Salpêtrière, Paris, F-75013, France

${ }^{7}$ Florey Institute of Neuroscience and Mental Health, University of Melbourne, Parkville, Victoria, 3010, Australia

${ }^{8}$ AP-HP, Hôpital de la Pitié-Salpêtrière, Department of Genetics, Paris, F-75013, France

The authors wish it to be known that, in their opinion, the last two authors should be regarded as joint Last Authors

* To whom correspondence should be addressed at: Institut du Cerveau et de la Moelle épinière, Hôpital de la Pitié-Salpêtrière, Inserm U1127, CNRS UMR 7225, Sorbonne Universités, Paris France, Email: olga.corti@upmc.fr 


\section{ABSTRACT}

Mutations of $L R R K 2$, encoding leucine-rich repeat kinase 2, are the leading cause of autosomal dominant Parkinson's disease (PD). The most frequent of these mutations, G2019S substitution, increases kinase activity, but it remains unclear how it causes PD. Recent studies suggest that LRRK2 modulates mitochondrial homeostasis. Mitochondrial dysfunction plays a key role in the pathogenesis of autosomal recessive PD forms linked to PARK2 and PINK1, encoding the cytosolic E3 ubiquitin-protein ligase Parkin and the mitochondrial kinase PINK1, which jointly regulate mitophagy.

We explored the role of LRRK2 and its kinase activity in PINK1/Parkin-dependent mitophagy. LRRK2 increased mitochondrial aggregation and attenuated mitochondrial clearance in cells coexpressing Parkin and exposed to the protonophore CCCP. FRET imaging microscopy showed that LRRK2 impaired the interactions between Parkin and Drp1 and their mitochondrial targets early in mitophagy. The inhibition of LRRK2 kinase activity by a "kinase-dead" LRRK2 mutation or with a pharmacological inhibitor (LRRK2-IN-1) restored these interactions. The monitoring of mitophagy in human primary fibroblasts with the novel dual-fluorescence mtRosella reporter and a new hypothermic shock paradigm revealed similar defects in PD patients with the G2019S LRRK2 substitution or PARK2 mutations relative to healthy subjects. This defect was restored by LRRK2-IN-1 treatment in LRRK2 patients only.

Our results suggest that PD forms due to LRRK2 and PARK2 mutations involve pathogenic mechanisms converging on PINK1/Parkin-dependent mitophagy. 


\section{INTRODUCTION}

Parkinson's disease (PD) is the second most common neurodegenerative disorder, characterized by the progressive loss of the dopaminergic neurons of the substantia nigra pars compacta. Most cases of PD are sporadic, but about $10 \%$ of the cases have a familial origin. Over the last two decades, disease-causing mutations with autosomal dominant (e.g. PARK1/SNCA, PARK8/LRRK2) or recessive (e.g. PARK2/Parkin, PARK6/PINK1) transmission have been identified in about 20 genes (1). Mutations in LRRK2 are the commonest cause of both familial and sporadic PD forms, with reported frequencies of 15$40 \%$, depending on the population studied (2-4). Mutations in PARK2 are the commonest cause of early-onset PD, with reported frequencies of 10-20\% worldwide (5).

Leucine-Rich Repeat Kinase 2 (LRRK2) is a large multidomain protein of the ROCO family, with both kinase and GTPase activities (6). The most frequent mutation of the gene encoding this protein leads to the G2019S substitution in the kinase domain and has been associated with an increase in kinase activity and with neurotoxicity in cell models (7-10). The precise function of LRRK2 remains unknown, but various roles in vesicle synthesis and trafficking (11), microtubule dynamics $(10,12,13)$, inflammation (14-16) and autophagy (17-20) have been described. LRRK2 has also been linked to the regulation of mitochondrial homeostasis. Several studies have shown that the G2019S substitution disturbs mitochondrial function, decreasing mitochondrial membrane potential, ATP levels and production, and inducing ROS imbalance, and changes in fusion/fission dynamics (21-24). In particular, several groups have reported a functional interaction with the mitochondrial fission-promoting dynamin-related protein 1 (Drp1) $(23,25-28)$.

Drp1-dependent mitochondrial fission occurs early in mitophagy and promotes the isolation of the damaged organelle from the healthy network (29). Two proteins encoded by genes 
implicated in autosomal recessive PD forms - the E3 ubiquitin protein ligase Parkin and the mitochondrial serine/threonine PINK1 — jointly regulate a mitophagy program triggered by mitochondrial depolarization (30-33). This process involves a sequence of events initiated by the accumulation of PINK1 close to the translocase of outer mitochondrial membrane (TOM complex), followed by the mitochondrial translocation and activation of Parkin, the ubiquitylation of a series of mitochondrial proteins and the recruitment of ubiquitin-binding autophagy adaptors and the autophagy machinery $(34,35)$. We have shown that PINK1 and Parkin cooperate in the recruitment of Drp1 to mitochondria during mitophagy (36).

We investigated the mechanisms by which LRRK2 affects mitochondrial function, by exploring its potential impact on PINK1/Parkin-dependent mitophagy and the effects of the G2019S substitution. Using cell lines and primary fibroblasts from PD patients, we showed that LRRK2 attenuated mitophagy, and that this effect was exacerbated by the G2019S substitution. We also provide evidence that LRRK2 interferes with protein-protein interactions involving Parkin and Drp1 on the outer mitochondrial membrane (OMM), and that these interactions, together with the defect in PINK1/Parkin-dependent mitophagy, can be rescued by the inhibition of LRRK2 kinase activity. 


\section{RESULTS}

\section{LRRK2 attenuates PINK1/Parkin-dependent mitophagy in a kinase-dependent manner}

We investigated the possible role of LRRK2 in PINK1/Parkin-dependent mitophagy in a model previously validated for the investigation of this process (36-38). In this model, Parkin is transiently overexpressed in COS7 cells. The cells are then treated with CCCP (carbonylcyanide m-chlorophenylhydrazone) and mitophagy is followed over time by quantifying two relevant parameters: (1) perinuclear mitochondrial aggregation, an early Parkin-dependent phenomenon preceding mitochondrial degradation (39); and (2) the clearance of mitochondrial markers. We investigated the impact of LRRK2 on mitophagy, by expressing Parkin alone or with full-length LRRK2. Under cotransfection conditions, exogenous Parkin was expressed in the vast majority of the LRRK2-immunopositive cells (95\%, data not shown). Moreover, western blots revealed that exogenous LRRK2 expression had no major impact on Parkin levels and that LRRK2 was undetectable in native cells (Supplementary Material, Fig. S1). We then analyzed the mitochondrial network after staining for a marker of the mitochondrial matrix (the $\beta$ subunit of the mitochondrial processing peptidase, PMPCB) or the outer mitochondrial membrane (the TOMM20 subunit of the translocase of outer mitochondrial membrane, data not shown) in cells immunopositive for Parkin (Parkin-only conditions) or for LRRK2 (cotransfection conditions). After $48 \mathrm{~h}$ of CCCP treatment, about $35 \%$ of the cells expressing Parkin alone had aggregated mitochondrial networks and $20 \%$ had lost all mitochondrial staining. In cells coexpressing LRRK2, the percentage of cells without mitochondrial staining was significantly smaller, but levels of mitochondrial aggregation were similar (Fig. 1A and B). As expected, no mitochondrial aggregation or clearance was observed in the absence of Parkin (Supplementary Material, Fig. S2 and data not shown), regardless of the presence or absence of LRRK2 (Fig. 1A and B). We then investigated the effect of LRRK2 kinase activity on the 
observed decrease in Parkin-dependent mitophagy, by expressing two LRRK2 variants with altered kinase activity: LRRK2-G2019S, reported to have higher kinase activity (7-10), and the kinase-dead LRRK2-D1994A variant (40-42) (Fig. 1C, Supplementary Material, Fig. S3). Western blot analysis revealed that the abundance of these variants was significantly lower than that of the normal LRRK2 protein in cells (Supplementary Material, Fig. S1). This was not as much due to differences in protein abundance in individual cells as it reflected lower proportions of cells expressing the mutant proteins, possibly linked to toxicity. For the analysis of mitophagy, we took into account only cells with similar levels of LRRK2 immunoreactivity. The proportion of cells with aggregated mitochondria was significantly higher following the expression of LRRK2-G2019S than with LRRK2, at $60 \%$ rather than 40\%. Moreover, the expression of LRRK2-G2019S further reduced mitochondrial clearance, by approximately 50\%. By contrast, LRRK2-D1994A had no effect on mitochondrial aggregation and tended to weaken the impact of LRRK2 on mitochondrial clearance (Fig. 1C, Supplementary Material, Fig. S3; $p$-value $<0.05$ versus Parkin-only condition compared to $p$ value $<0.001$ versus Parkin-only condition for LRRK2). These results suggest that LRRK2 attenuates Parkin-dependent mitophagy in a kinase-dependent manner, through effects on an early step in this process associated with the formation of mitochondrial aggregates. We explored this relationship further, by performing a kinetic analysis of mitochondrial aggregation in cells expressing Parkin alone (Parkin-only conditions) or together with LRRK2, LRRK2-G2019S or LRRK2-D1994A (Fig. 1D). As expected from our previous experience (36-38), the proportion of cells with mitochondrial aggregates progressively increased in the presence of overexpressed Parkin, reaching a plateau after $8 \mathrm{~h}$ of CCCP treatment. A similar progression was observed in cells coexpressing the kinase-dead LRRK2D1994A variant. By contrast, the proportion of cells with aggregated mitochondria at each of the time points analyzed was significantly higher in cells coexpressing LRRK2, and higher 
still in cells coexpressing LRRK2-G2019S. Cell treatment with the well-known LRRK2 kinase inhibitor, LRRK2-IN-1 (43) $(5 \mu \mathrm{M})$, normalized the aggregation profile in cells coexpressing LRRK2 and significantly decreased the proportion of cells with aggregated mitochondria in cells coexpressing LRRK2-G2019S (Fig. 1E). By contrast, LRRK2-IN-1 had no impact on the aggregation profile in cells expressing Parkin alone or together with LRRK2-D1994A (Fig. 1F). These observations highlight the role of LRRK2 kinase activity in mitochondrial aggregation, an early phenomenon in mitophagy occurring before mitochondrial clearance.

\section{LRRK2 kinase activity interferes with protein-protein interactions involving Parkin and}

\section{Drp1 on mitochondria}

In previous studies, we used Förster resonance energy transfer (FRET) microscopy with the acceptor photobleaching paradigm to monitor protein-protein interactions on the OMM (36, 37). These studies showed that Parkin is recruited to the mitochondria, in close proximity to PINK1 and the TOM complex, early in mitophagy, and that it cooperates with PINK1 in the recruitment to mitochondria of the dynamin-related GTPase Drp1, which is essential for efficient mitophagy $(29,36,44)$. All these interactions, detected upon mitochondrial depolarization, were strictly dependent on the presence of PINK1, and were affected by Parkinson's disease-linked PARK2 mutations $(36,37)$. We therefore used this method to explore the impact of LRRK2 on the molecular proximity of Parkin to TOM complex subunits, and of Parkin to Drp1, following CCCP treatment. As previously reported, we detected FRET between the TOMM40 and TOMM70A subunits of the TOM complex and Parkin (Fig. 2A), and between Drp1 and Parkin (Fig. 2D and E) (37). By contrast, no FRET was detected between TOMM20, TOMM22, VDAC1 and Parkin (Fig. 2A). The overexpression of LRRK2 and LRRK2-G2019S abolished FRET between the 
abovementioned donor/acceptor pairs, whereas FRET was preserved with the kinase-dead LRRK2-D1994A variant (Fig. 2A). The treatment of cells expressing LRRK2 or LRRK2G2019S with LRRK2-IN-1 restored FRET efficiencies between the investigated protein pairs (Fig. 2B-E, Supplementary Material, Fig. S4). We explored the impact of LRRK2 on the mitochondrial recruitment of Drp1 directly, by investigating the interaction between Drp1 and its mitochondrial receptor, MiD51, which we have previously shown to be required for Parkin-dependent mitophagy (36). FRET was detected between Drp1 and MiD51 in cells treated with CCCP, but was abolished in cells overexpressing LRRK2 or LRRK2-G2019S. Again, the Drp1-MiD51 interaction was preserved in the presence of LRRK2-D1994A and restored by LRRK2-IN-1 treatment in cells expressing active LRRK2 variants (Fig. 2F and $\mathrm{G})$.

Overall, these results suggest that the kinase activity of LRRK2 disrupts protein-protein interactions on the outer mitochondrial membrane occurring early in PINK1/Parkin-dependent mitophagy, including the recruitment of Drp1 to its receptor, MiD51.

\section{A new model for studying Parkin-dependent mitophagy in human fibroblasts}

We evaluated the relevance of our findings, by comparing the efficiency of mitophagy in primary fibroblasts from healthy controls and patients carrying the activating G2019S LRRK2 mutation or PARK2 mutations. Our analysis of the mitophagy triggered by CCCP in human fibroblasts with endogenous levels of Parkin led to inconsistent results. Specifically, the severe fragmentation of the mitochondrial network made it impossible to obtain reliable estimates of the modest decreases in the abundance of specific mitochondrial markers. Previous studies have shown that autophagy can be stimulated by a heat shock (45-47). We therefore developed a new mitophagy paradigm based on induction by heat stress, to avoid the 
massive mitochondrial depolarization triggered by CCCP. We also used a mitophagy reporter consisting of a $\mathrm{pH}$-stable DsRed.T4 fused to a green fluorescent protein sensitive to acidic conditions (pHluorin) and targeted to mitochondria by a classical N-terminal mitochondrial targeting signal (mtRosella) for the quantitative assessment of mitophagy in living cells. Rosella constructs have been successfully used in the monitoring of autophagy-dependent processes, including mitophagy, in yeast and Caenorhabditis elegans, based on estimation of the ratio of the red-only signal, reflecting the presence of the reporter in the acidic lysosomal compartment, to green/red double fluorescence $(48,49)$. We here report the first application of this system to mammalian cells. Fibroblasts from control individuals overexpressing mtRosella were shifted to either $42^{\circ} \mathrm{C}$ or $24^{\circ} \mathrm{C}$ for 45 minutes and then returned to $37^{\circ} \mathrm{C}$ for 45 minutes (Fig. 3A and B). The shift to $42^{\circ} \mathrm{C}$ resulted in an overall compaction of the mitochondrial network relative to basal conditions (cells maintained at $37^{\circ} \mathrm{C}$ ), with no change in the red-only signal. By contrast, the shift to $24^{\circ} \mathrm{C}$ led to an increase in the abundance of mitochondrial vesicles with red-only fluorescence, resulting in a doubling of mean red-only fluorescence relative to basal conditions. This increase was abolished following treatment with the autophagy inhibitor 3-methyladenine (3-MA) (Fig. 3C), and in cells silenced for ATG5 (Fig. 3D), Parkin (Fig. 4A and B) or PINK1 (Fig. 4C and D), indicating that it was related to PINK1/Parkin-dependent mitophagy. Moreover, the red-only fluorescent mitochondrial vesicles corresponded to mitochondria engulfed by lysosomes, as shown by confocal microscope analyses of co-localization with the lysosomal LysoTracker dye (Supplementary Material, Fig. S5). More than $90 \%$ of the red-only signal colocalized with Lysotracker fluorescence, whereas no more than $15 \%$ of the green fluorescent mitochondrial staining colocalized with lysosomes. Thus, shifting cells to $24^{\circ} \mathrm{C}$ triggers Parkin-dependent mitophagy in human fibroblasts. 


\section{Parkin-dependent mitophagy is impaired in fibroblasts from PD patients}

We then used the shift to $24^{\circ} \mathrm{C}$ to investigate Parkin-dependent mitophagy in fibroblasts from control subjects and PD patients with the G2019S LRRK2 mutation, or various compound heterozygous combinations of PARK2 mutations (Table 1). We previously showed that Parkin was detectable in human fibroblasts (50). Western blot analysis showed that LRRK2 was present in cells from each category of subjects (Supplementary Material, Fig. S6). The mtRosella reporter was expressed following transient transfection, and mitophagy was investigated in four independent sessions, each including a different fibroblast line from each category of subjects (Supplementary Material, Table S1 and Table 1). As expected, the overall analysis of these experiments revealed that, in control fibroblasts, the shift to $24^{\circ} \mathrm{C}$ led to the appearance of numerous mitochondrial vesicles with red-only fluorescence. By contrast, red-only fluorescence was much less abundant in fibroblasts from patients with PARK2 mutations and carriers of the G2019S LRRK2 mutation (Fig. 5A and C). The levels of red-only fluorescence in cells from patients were similar to those in control cells maintained at $37^{\circ} \mathrm{C}$ (Fig. 3B), suggesting that there was no effect on basal mitophagy. An impaired induction of mitophagy was observed for all patient lines relative to any control line, demonstrating the robustness of these results (Supplementary Material, Table S1). We evaluated the dependence of the apparent impairment of mitophagy induction in LRRK2 patients on the kinase activity of the protein, as suggested by our results in COS7 cells, by treating the cells with LRRK2-IN-1 (Fig. 5B and C). LRRK2-IN-1 restored Parkin-dependent mitophagy in fibroblasts with the G2019S LRRK2 mutation, but not in fibroblasts with PARK2 mutations. The inhibition of LRRK2 kinase activity had no effect on mitophagy induction in fibroblasts from control individuals. 
Our results for COS7 cells suggested that LRRK2 overactivity disturbs the mitochondrial recruitment of Drp1 preceding mitophagy. We therefore investigated whether rescue of the mitophagy defect by LRRK2-IN-1 in fibroblasts from LRRK2-G2019S patients was dependent on functional Drp1. We transfected fibroblasts from LRRK2-G2019S patients with the mtRosella construct together with an empty vector or vectors encoding the dominant negative Drp1-K38A variant or functional Drp1 (Fig. 6A and B). We evaluated the impact of LRRK2-IN-1 on the restoration of mitophagy following a shift to $24^{\circ} \mathrm{C}$ in these conditions. As previously observed, LRRK2-IN-1 restored mitophagy in fibroblasts from LRRK2G2019S patients transfected with empty or functional Drp1 vectors. By contrast, mitophagy induction was not rescued in cells expressing Drp1K38A (Fig. 6A and B). Overexpression of Drp1 in cells from LRRK2-G2019S patients did not rescue the mitophagy defect per se, stuggesting that this approach does not restore the mitochondrial recruitment of Drp1. For confirmation of the requirement of Drp1 to rescue mitophagy induction in LRRK2-G2019S fibroblasts, we treated the cells with the cell-permeable Drp1 inhibitor Mdivi-1 (51); again, no correction of the mitophagy defect by LRRK2-IN-1 was observed (Fig. 6C and D). These results suggest that the impairment of Parkin-dependent mitophagy in LRRK2-G2019S patients is at least partly mediated by Drp1. To gain insight into possible mechanisms underlying Drp1 dysregulation by LRRK2-G2019S, we examined intracellular levels of Drp1 or Drp1 forms phosphorylated on serine 616 or serine 637, known respectively to promote mitochondrial translocation and fission (52) or sequestration of the protein in the cytosol (53), in fibroblasts from LRRK2-G2019S patients and control subjects (Supplementary Material, Fig. S7). As a control, we also analyzed levels of the profusion GTPase Mfn2. The abundance of total Drp1, Drp1 phosphorylated on serine 616 and Mfn2 were similar in cells from patients and controls, both under basal conditions and following a shift to $24^{\circ} \mathrm{C}$. Drp 1 phosphorylated on serine 637 was undetectable in these experimental conditions. 


\section{DISCUSSION}

We explored the involvement of LRRK2 in PINK1/Parkin-dependent mitophagy in two complementary model systems: a classical model based on the overexpression of Parkin and the coexpression of LRRK2 variants in a human cell line, and induction of mitochondrial dysfunction using the mitochondrial uncoupler CCCP; and an original model with physiological expression of Parkin and LRRK2 variants, based on the use of primary human fibroblasts from control individuals or PD patients, and thermal stress to trigger mitophagy. A combination of approaches were used to explore mitophagy in these models, including analysis of mitochondrial network aggregation and clearance, investigation of key proteinprotein interactions established during the early phases of the process, and the use of the novel sensitive dual-fluorescence mitophagy reporter, mtRosella. We provide several lines of evidence consistent with a role for LRRK2 kinase hyperactivity in downregulating PINK1/Parkin-dependent mitophagy. First, the overexpression of kinase-active LRRK2 accentuated the perinuclear accumulation of damaged mitochondria and attenuated their Parkin-dependent clearance triggered by CCCP. These effects were exacerbated by the expression of the kinase hyperactive LRRK2-G2019S variant, and mitigated or abolished by expression of the kinase inactive LRRK2-D1994A variant, or treatement of the cells with a pharmacological LRRK2 kinase inhibitor. Second, LRRK2 interfered with the recruitment of Parkin and the fission-promoting GTPase Drp1 to their respective interactors on the OMM, the TOM complex and the receptor MiD51. Again, this effect was abolished by genetic and pharmacological approaches aimed at inhibiting LRRK2 kinase activity. Third, the use of mtRosella, revealed that LRRK2 overactivation compromised the induction of PINK1/Parkindependent mitophagy in fibroblasts from PD patients carrying the LRRK2-G2019S substitution. This defect was similar to that observed in patients with compound heterozygous 
PARK2 mutations, and was rescued by a LRRK2 kinase inhibitor. Finally, our results suggest that the effect of LRRK2 kinase hyperactivity on PINK1/Parkin-dependent mitophagy is at least in part mediated by Drp1.

Previous studies have already highlighted a functional interplay between LRRK2 and Drp1, albeit with inconsistent results $(54)(23,25-28)$. Consistent with our findings, the inhibition of LRRK2 kinase activity has been shown to promote the association of Drp1 with mitochondria and mitochondrial fission (55), indicating that impairment of the mitochondrial translocation of Drp1 may well underlie the mitophagy defect in cells from patients with the G2019S substitution. Consistent with this possibility, other authors have reported changes in mitochondrial morphology compatible with fission arrest in LRRK2-G2019S knock-in mice and fibroblasts from patients carrying the newly discovered LRRK2 substitution E193K (28, 56). In mice, these alterations were accompanied by a decrease in levels of Drp1 phosphorylated on serine 616 (52). The specific mechanisms by which LRRK2 regulates Drp1 to inhibit mitochondria fission before mitophagy remain to be determined. It has been suggested that LRRK2 phosphorylates Drp1 directly on the serine 637 residue, thereby preventing translocation of the protein from the cytosol to the OMM (53). We did not detect changes to the phosphorylation state of serine 616 or serine 637 under basal conditions or following thermal stress in total lysates from fibroblasts derived from carriers of the G2019S mutation compared to control cells. This does not exclude a possible impact of LRRK2G2019S on the mitochondrial pool of Drp1. Such an impact may be indirect and possibly involve other posttranslational modifications known to regulate the mitochondrial association of Drp1 (52).

The role of LRRK2 in macroautophagy has also been intensively investigated in previous studies (54), several of which concluded that its effect is proautophagic (10, 17, 19, 57-59), and others reporting an inhibitory effect $(20,60,61)$ or no effect $(62)$. Previous attempts to 
address the impact of LRRK2 on basal mitophagy $(52,58)$, which is unaffected by PINK1 and Parkin $(63,64)$ showed opposing effects, whereas a single study assessing toxin-induced mitophagy in fibroblasts from patients with sporadic and LRRK2-linked PD, using colocalization of TOMM20 and the lysosomal marker LAMP1 as a supposed "mitophagy index" was inconclusive (65). Only one previous study investigated, in detail, the direct impact of LRRK2 on PINK1/Parkin-dependent mitophagy, and its findings are consistent with our observations (66). This study showed that axonal mitophagy was delayed in induced pluripotent stem cell (iPSC)-derived neurons from patients with sporadic PD and patients with the LRRK2-G2019S mutation, through a combination of approaches, including the colocalization of mitochondria with LC3 or optineurin and the use of the ratiometric mitophagy reporter mt-Keima. This defect was linked to a mechanism converging on the outer mitochondrial membrane protein Miro, a GTPase that anchors mitochondria via molecular motors to the microtubule network and is known to be cleared by the joint action of PINK1 and Parkin before mitophagy. The authors showed that LRRK2 facilitated mitochondrial arrest by promoting the removal of Miro from damaged mitochondria, an effect that was disrupted by the G2019S substitution. Mitochondrial fission and detachment from microtubules are early steps in mitophagy that are thought to require coordination for engulfment of the damaged organelle by the autophagosome. An impact of LRRK2 on mitochondrial arrest is, therefore, not incompatible with an effect on mitochondrial fission during mitophagy, and might be particularly relevant to the efficiency of mitophagy, particularly in highly polarized cells such as neurons.

Interestingly, the molecular events that initiate mitophagy are integrated by a physical platform corresponding to the interface between the endoplasmic reticulum (ER) and mitochondria that has been reported to be regulated by PINK1 and Parkin (50, 67-69). ER tubules mark sites of Drp1 recruitment and mitochondrial fission (70), and mitochondria are 
progressively degraded at the ER-mitochondrion interface (71). PINK1 and Parkin promote the degradation of the profusion GTPase Mfn2, which tethers the mitochondria to the ER in mammalian cells $(72,73)$. Subsequent dissociation of the mitochondria from the ER is essential for efficient mitophagy (69). The Miro GTPase Gem1 is an integral component of the ER-mitochondrion interface in yeast, and this role may have been conserved throughout evolution (74), in which case, PINK1/Parkin-dependent Miro degradation or LRRK2mediated removal from mitochondria during mitophagy may also contribute to organelle separation. Remarkably, mitochondria-lysosome contacts have also been involved in the regulation of Drp1-dependent mitochondrial fission events at sites marked by ER tubules, through GTP hydrolysis by the small Rab GTPase, Rab7, driven by the Rab7 GTPaseactivating protein, TBC1D15 (75). Rab7 and TBC1D15 also regulate autophagosme biogenesis during PINK1/Parkin-dependent mitophagy (76), and LRRK2 and its substrate Rab GTPases are recruited to overloaded lysosome to maintain their homeostasis (77).

These observations suggest that, like PINK1 and Parkin, LRRK2 may modulate mitophagy by acting on molecular actors involved in various steps of mitochondrial degradation in a dynamically controlled manner. Further studies are required to explore this hypothesis in greater depth, and to determine the extent to which the regulation of these molecular events corresponds to a physiological role of LRRK2, rather than a gain of function linked to PDcausing mutations. Notably, evidence for LRRK2 hyperactivation was recently provided in brain tissue from patients with idiopathic PD and in two distinct rat models of PD (78). This defect was linked to a pathological cascade initiated by oxidative stress, and involving autophosphorylation of LRRK2 and secondary accumulation of signs of mitochondrial and lysosomal dysfunction. Therefore, LRRK2 kinase inhibition may be considered as a potential therapeutic approach for correcting mitochondrial and autophagy defects, possibily not only in patients with LRRK2 mutations but also, more generally, in idiopathic PD. 



\section{MATERIALS AND METHODS}

\section{Human fibroblasts}

Human fibroblasts were obtained from skin biopsy specimens obtained from patients with the G2019S LRRK2 substitution or with PARK2 mutations and healthy individuals, at the Clinical Investigation Center for Neurosciences at Pitié-Salpêtrière hospital. All patients signed an informed consent before the procedure, and the study was approved by a local ethics committee and the regulatory authorities. Patients were screened for PARK2 mutations by exon dosage and bidirectional Sanger sequencing of the entire coding sequence, as previously described (79). Patients were screened for the LRRK2-G2019S mutation in Taqman assays, with confirmation by bidirectional Sanger sequencing for those testing positive (80).

\section{Plasmids, pharmacological agents, treatments and antibodies}

The following plasmids were used for transfection: pcDNA3-HA, pcDNA-HA-Parkin, pDEST53-GFP-LRRK2/LRRK2-G2019S (full-length proteins) (9) and pDEST53-GFPLRRK2-D1994A (obtained from pDEST53-LRRK2-GFP by site-directed mutagenesis), pCB6-Myc-Drp1 and pCB6-Myc-Drp1-K38A (provided by M. Rojo). The pCINeo-mtRosella vector, encoding a pH-stable DsRed.T4 fused to a green fluorescent protein (pHluorin) linked to the COX VIII N-terminal mitochondrial targeting signal, was designed by Carlos Rosado (Mark Prescott's laboratory, Monash University, Australia). RNA interference approaches involved: ATG5 siRNA (Qiagen, SI02655310), Parkin siRNA (Invitrogen, HSS107593), PINK1 siRNA (Thermo Fisher, HSS127945) and all-star siRNA (control; Qiagen, 1027281), all used at a concentration of $100 \mathrm{nM}$.

The pharmacological agents used were: $10 \mu \mathrm{M}$ CCCP (Sigma, C2759) for $48 \mathrm{~h}$ (mitophagy analysis) or for $6 \mathrm{~h}$ (FRET experiments), $10 \mathrm{mM}$ 3-methyladenine (3-MA, Sigma, M9281) for 
$3 \mathrm{~h}$ (Rosella experiments), $5 \mu \mathrm{M}$ LRRK2-IN-1 (Millipore, 438193) for 0 to $12 \mathrm{~h}$ (mitochondrial aggregation kinetics), $2 \mathrm{~h}$ (Rosella experiments) or $6 \mathrm{~h}$ (FRET experiments) and $50 \mu \mathrm{M}$ Mdivi-1 (Sigma, MO199) for $90 \mathrm{~min}$ (Rosella experiments). Primary fibroblasts were exposed to heat shock by shifting them from $37^{\circ} \mathrm{C}$ to $24^{\circ} \mathrm{C}$ or $42^{\circ} \mathrm{C}$ for $45 \mathrm{~min}$. They were then returned to $37^{\circ} \mathrm{C}$ for 45 min before analysis.

The primary antibodies used for western blotting or for immunocytochemistry and FRET experiments are listed in Tables 2 and 3, respectively.

\section{Cell culture and transfection}

COS7 cells were maintained in DMEM GlutaMAX ${ }^{\mathrm{TM}}$ (Dulbecco's modified Eagle's medium; Gibco, 10566016) supplemented with 10\% (v/v) fetal bovine serum (Gibco, 10270106) and $1 \%(\mathrm{v} / \mathrm{v})$ antibiotics (penicillin and streptomycin, Invitrogen, 25030024$)$ at $37^{\circ} \mathrm{C}$, under a humidified atmosphere containing $5 \% \mathrm{CO}_{2}$. Cells were plated at a density of 30,000 (FRET experiment) or 40,000 cells per well (mitophagy analysis) in 24-well culture plates (Corning, 3527). They were added to the wells on 14-mm glass coverslips precoated with poly-D-lysine (PDL, Sigma, P7886; $250 \mu \mathrm{g} / \mathrm{ml}$ ). After incubation at $37^{\circ} \mathrm{C}$ for 24 hours, the cells were transfected with $1 \mu \mathrm{g}$ of plasmid per well in the presence of DMRIE-C agent (Invitrogen, 10459-014) in Opti-MEM ${ }^{\mathrm{TM}}$ medium (Thermo Fisher Scientific, 31985070) for 5 hours, according to the manufacturer's instructions. In cotransfections, pcDNA3-HA-Parkin and pDEST53-GFP-LRRK2 vectors were used at a ratio of 1:9 by weight. At the end of the fivehour transfection period, the transfection medium was removed and the cells were incubated overnight with complete DMEM GlutaMAX ${ }^{\mathrm{TM}}$ at $37^{\circ} \mathrm{C}$, under an atmosphere containing $5 \%$ $\mathrm{CO}_{2}$. The cells were exposed to pharmacological agents (see above) 24 hours later. They were then fixed by incubation in 4\% paraformaldehyde (PFA, Sigma, 252549) for 20 minutes, washed three times with phosphate-buffered saline (PBS) and stored at $4^{\circ} \mathrm{C}$. 
Human fibroblasts were maintained at $80 \%$ confluence in complete DMEM GlutaMAX ${ }^{\mathrm{TM}}$ medium. Fibroblasts were transfected with the following plasmids: $1 \mu \mathrm{g}$ of mtRosella plasmid alone, with other plasmids $(1 \mu \mathrm{g})$ or with siRNAs $(100 \mathrm{nM})$, per 20,000 cells. Cells were transfected by electroporation ( 2 pulses, $20 \mathrm{~ms}$ and $1200 \mathrm{~V}$ ) with the Neon ${ }^{\circledR}$ Transfection System (Thermo Fisher Scientific, MPK10096) and plated in Ibidi glass-bottomed eight-well chambers (Biovalley, 80827; 20,000 cells per well) or in six-well plates (TPP, 92006; 400,000 cells per well), in phenol red-free medium supplemented with $10 \%(\mathrm{v} / \mathrm{v})$ FBS and $1 \%(\mathrm{v} / \mathrm{v})$ glutamine. The medium was replaced after $24 \mathrm{~h}$, and analyses were performed $72 \mathrm{~h}$ after transfection.

\section{Immunocytochemistry}

Cells were processed with standard immunocytochemical techniques. In brief, cells were permeabilized by incubation in $0.2 \%$ Triton X-100 in PBS for 10 minutes and nonspecific binding sites were blocked by incubation for 1 hour at room temperature with $10 \%$ normal goat serum (NGS, Thermo Fisher Scientific, 1610064). Cells were incubated with primary antibodies diluted in PBS supplemented with $2 \%$ NGS overnight at $4{ }^{\circ} \mathrm{C}$, rinsed and incubated with secondary antibodies in PBS supplemented with 2\% NGS at room temperature for $1 \mathrm{~h}$. The coverslips holding the cells were then mounted in ProLong ${ }^{\circledR}$ Gold Antifade Reagent (Thermo Fisher Scientific, P36930) on glass microscope slides. For the analysis of mitophagy in COS7 cells, we performed dual labeling for Parkin (Parkin-only conditions) or LRRK2 (Parkin + LRRK2 conditions) and either TOMM20 or PMPCB. For FRET experiments, the impact of LRRK2 variants on specific protein-protein interactions was assessed by triple labeling for LRRK2 and the specific donor/acceptor pairs.

\section{Epifluorescence and confocal microscopy}


We analyzed the effects of LRRK2 on Parkin-dependent mitophagy, by imaging COS7 cells with a Nikon Eclipse Ti-U M7 epifluorescence microscope equipped with HCImage software (20 x objective). We analyzed at least 60 cells immunopositive for Parkin (Parkin-only condition) or for LRRK2 or LRRK2 variants (Parkin + LRRK2 conditions) per condition, in each of at least three independent experiments, checking for the presence of aggregated mitochondria or the absence of mitochondrial staining. The effects of different LRRK2 variants were investigated in cells with similar fluorescence immunostaining intensities for the N-terminal GFP moiety encoded by the GFP-LRRK2 constructs. For FRET experiments, we selected cells immunopositive for LRRK2 (Alexa Fluor ${ }^{\circledR} 405$ blue fluorescence) and containing mitochondrial aggregates reflecting the presence of Parkin. Images of donor (Alexa Fluor ${ }^{\circledR} 488$ green) and acceptor (Alexa Fluor ${ }^{\circledR} 568$ red) fluorescence (see Table 3) were acquired from a region of interest (ROI) before and after photobleaching, with an Olympus FluoView FV-100 microscope driven by Olympus FV-1000 3.1 software (63 x oil immersion objective, N. A. 1.35). Care was taken to investigate the effects of different LRRK2 variants in cells with similar immunofluorescence intensities for these proteins. Before analyzing the effects of LRRK2 on specific protein-protein interactions, we checked that the Alexa Fluor ${ }^{\circledR} 405$ blue fluorescence did not itself interfere with FRET efficiencies, by comparing specific donor/acceptor pairs in cells co-immunolabeled for GAPDH or tubulin, using Alexa Fluor ${ }^{\circledR}$ 405-coupled secondary antibodies (data not shown). We analyzed seven to 12 cells per condition, in each of at least three independent experiments. We used ImageJ software after photobleaching to correct images for the decrease in fluorescence intensity due to the acquisition process, and quantification was performed as previously described (37): \% FRET $=\left[I_{D}\right.$ postbleach $-I_{D}$ prebleach $) / I_{D}$ postbleach $]_{\text {ROI. }}$ For the analysis of mitophagy based on the mtRosella construct in human fibroblasts, cells were captured at $37^{\circ} \mathrm{C}$ with a Spinning disk CSU-X1 confocal microscope (Leica) equipped with a chamber with controlled 
temperature and $\mathrm{CO}_{2}$ concentration, for live cell imaging driven by MetaMorph software (63 $\mathrm{x}$ immersion oil objective, N.A. 1.4). We analyzed 12-20 cells per condition in each of at least three independent experiments. For comparative analyses of mitophagy in fibroblasts from control subjects and PD patients, several independent experiments were run, each including cells from a control, and at least one category of patients (Supplementary Material, Table S1). Images were deconvoluted with Huygens Professional software and analyzed with Fiji Software to quantify the ratio of the mean area of red-only fluorescence to total red fluorescence per cell (\%).

\section{Immunoblotting}

After $24 \mathrm{~h}$, cells were washed, collected and lysed directly in 1 x sample buffer with DTT (dithiothreitol), protease inhibitors (Thermo Fisher Scientific, 1821281) and phosphatase inhibitors (Thermo Fisher Scientific, 1821279). Proteins were quantified with the Pierce TM BCA protein Assay (Thermo Fisher Scientific, 23225), with absorbance readings at $562 \mathrm{~nm}$. Whole-cell lysates were heated at $95^{\circ} \mathrm{C}$ for $5 \mathrm{~min}$, separated by electrophoresis in a $4-20 \%$ agarose Tris/glycine gel (Mini-PROTEAN ${ }^{\circledR}$ TGX $^{\mathrm{TM}}$ Precast Gels, BioRad, 4561094), and the proteins were then transferred onto nitrocellulose membrane by wet electroblotting (Amersham Protran, $0.45 \mu \mathrm{m}$ pores; GE Healthcare, 10600002). The membrane was incubated in 5\% non-fat dry milk powder in PBS to block nonspecific binding sites, then overnight at $4{ }^{\circ} \mathrm{C}$ with primary antibody in $5 \%$ non-fat dry milk powder in PBS, and for $1 \mathrm{~h}$ at room temperature with horseradish peroxidase (HRP)-conjugated secondary antibody (1:50000) in $1 \%$ non-fat dry milk powder in PBS. Proteins were visualized by enhanced chemiluminescence (ECL SuperSignal ${ }^{\mathrm{TM}}$ West Dura (Thermo Fisher Scientific, 34075) on film (ECL, Amersham Hyperfilm, 28906837). Signals were quantified with MultiGauge software. 


\section{Statistical analyses}

Statistical analysis was performed with Prism 7 (Graph Pad software). The results are expressed as means $\pm \mathrm{SD}$, with the exception of the kinetics analyses based on the evaluation of an interaction factor (time and different conditions). One- or two-way ANOVA followed by Tukey's multiple comparisons post-hoc test was used to evaluate the differences between groups, except for the analysis of the effects of 3-MA treatment, ATG5 siRNA, Parkin siRNA and PINK1 siRNA on mtRosella responses in fibroblasts, for which paired Student's $t$-tests were used. Values of $p<0.05$ were considered significant. 


\section{SUPPLEMENTARY MATERIAL}

Supplementary Material is available at HMG online

\section{ACKNOWLEDGEMENTS}

We would like to thank S. Forlani (DNA and Cell Bank, Hôpital de la Pitié-Salpêtrière) for human cell collection and banking. We also thank S. Bardien for providing LRRK2/LRRK2G2019S vectors (Stellenbosch University, South Africa). We are grateful to S. Fouquet for providing us with access to the imaging facilities at Institut de la Vision.

Conflict of interest statement. J.C. C. received consultant honoraria from Theranexus, BrainEver, Air Liquide, BMS, Pfizer, Zambon, Amarantus, research grants from Actelion, Ipsen, Clevexel and Sanofi-Aventis. O.C. received research grants from Servier Laboratories and Sanofi-Aventis. Ipsen Laboratories funded part of the work in this study.

\section{FUNDING}

This work was supported by funding from INSERM, Ipsen Laboratories, Association France Parkinson, the Innovative Medicines Initiative Joint Undertaking under grant agreement no. 115568, including financial contributions from the European Union Seventh Framework Programme (FP7/2007-2013), EFPIA and "Investissements d'Avenir" ANR-10-IAIHU-06. F.B. received financial support from Association Nationale Recherche Technologie and Association France Parkinson. Y.S.S. received support from the International Union of Biochemistry and Molecular Biology (IUBMB, Wood-Whelan Research Fellowhsip). 


\section{REFERENCES}

1. Deng,H., Wang,P. and Jankovic,J. (2017) The genetics of Parkinson disease. Ageing Res. Rev., 42, $72-85$.

2. Nichols,W.C., Pankratz,N., Hernandez,D., Paisán-Ruíz,C., Jain,S., Halter,C.A., Michaels,V.E., Reed,T., Rudolph,A., Shults,C.W., et al. (2005) Genetic screening for a single common LRRK2 mutation in familial Parkinson's disease. Lancet Lond. Engl., 365, 410-412.

3. Gilks,W.P., Abou-Sleiman,P.M., Gandhi,S., Jain,S., Singleton,A., Lees,A.J., Shaw,K., Bhatia,K.P., Bonifati,V., Quinn,N.P., et al. (2005) A common LRRK2 mutation in idiopathic Parkinson's disease. Lancet Lond. Engl., 365, 415-416.

4. Lesage,S., Patin,E., Condroyer,C., Leutenegger,A.-L., Lohmann,E., Giladi,N., Bar-Shira,A., Belarbi,S., Hecham,N., Pollak,P., et al. (2010) Parkinson's disease-related LRRK2 G2019S mutation results from independent mutational events in humans. Hum. Mol. Genet., 19, 19982004.

5. Corti,O., Lesage,S. and Brice,A. (2011) What genetics tells us about the causes and mechanisms of Parkinson's disease. Physiol. Rev., 91, 1161-1218.

6. Blanca Ramírez,M., Madero-Perez,J., Rivero-Rios,P., Martinez-Salvador,M., Lara Ordonez,A.J., Fernandez,B., Fdez,E. and Hilfiker,S. (2017) LRRK2 and Parkinson's Disease: From Lack of Structure to Gain of Function. Curr. Protein Pept. Sci., 18, 677-686.

7. West,A.B., Moore,D.J., Biskup,S., Bugayenko,A., Smith,W.W., Ross,C.A., Dawson,V.L. and Dawson,T.M. (2005) Parkinson's disease-associated mutations in leucine-rich repeat kinase 2 augment kinase activity. Proc. Natl. Acad. Sci. U. S. A., 102, 16842-16847.

8. Smith,W.W., Pei,Z., Jiang,H., Dawson,V.L., Dawson,T.M. and Ross,C.A. (2006) Kinase activity of mutant LRRK2 mediates neuronal toxicity. Nat. Neurosci., 9, 1231-1233.

9. Greggio,E., Jain,S., Kingsbury,A., Bandopadhyay,R., Lewis,P., Kaganovich,A., van der Brug,M.P., Beilina,A., Blackinton,J., Thomas,K.J., et al. (2006) Kinase activity is required for the toxic effects of mutant LRRK2/dardarin. Neurobiol. Dis., 23, 329-341.

10. MacLeod,D., Dowman,J., Hammond,R., Leete,T., Inoue,K. and Abeliovich,A. (2006) The familial Parkinsonism gene LRRK2 regulates neurite process morphology. Neuron, 52, 587-593.

11. Shin,N., Jeong,H., Kwon,J., Heo,H.Y., Kwon,J.J., Yun,H.J., Kim,C.-H., Han,B.S., Tong,Y., Shen,J., et al. (2008) LRRK2 regulates synaptic vesicle endocytosis. Exp. Cell Res., 314, 2055-2065.

12. Gandhi,P.N., Wang,X., Zhu,X., Chen,S.G. and Wilson-Delfosse,A.L. (2008) The Roc domain of leucine-rich repeat kinase 2 is sufficient for interaction with microtubules. J. Neurosci. Res., 86, 1711-1720.

13. Gillardon,F. (2009) Leucine-rich repeat kinase 2 phosphorylates brain tubulin-beta isoforms and modulates microtubule stability--a point of convergence in parkinsonian neurodegeneration? J. Neurochem., 110, 1514-1522.

14. Hakimi,M., Selvanantham,T., Swinton,E., Padmore,R.F., Tong,Y., Kabbach,G., Venderova,K., Girardin,S.E., Bulman,D.E., Scherzer,C.R., et al. (2011) Parkinson's disease-linked LRRK2 is expressed in circulating and tissue immune cells and upregulated following recognition of microbial structures. J. Neural Transm. Vienna Austria 1996, 118, 795-808. 
15. Thévenet,J., Pescini Gobert,R., Hooft van Huijsduijnen,R., Wiessner,C. and Sagot,Y.J. (2011) Regulation of LRRK2 expression points to a functional role in human monocyte maturation. PloS One, 6, e21519.

16. Gillardon,F., Schmid,R. and Draheim,H. (2012) Parkinson's disease-linked leucine-rich repeat kinase $2(\mathrm{R} 1441 \mathrm{G})$ mutation increases proinflammatory cytokine release from activated primary microglial cells and resultant neurotoxicity. Neuroscience, 208, 41-48.

17. Plowey,E.D., Cherra,S.J., Liu,Y.-J. and Chu,C.T. (2008) Role of autophagy in G2019S-LRRK2associated neurite shortening in differentiated SH-SY5Y cells. J. Neurochem., 105, 10481056.

18. Tong,Y., Giaime,E., Yamaguchi,H., Ichimura,T., Liu,Y., Si,H., Cai,H., Bonventre,J.V. and Shen,J. (2012) Loss of leucine-rich repeat kinase 2 causes age-dependent bi-phasic alterations of the autophagy pathway. Mol. Neurodegener., 7, 2.

19. Bravo-San Pedro,J.M., Niso-Santano,M., Gómez-Sánchez,R., Pizarro-Estrella,E., AiastuiPujana,A., Gorostidi,A., Climent,V., López de Maturana,R., Sanchez-Pernaute,R., López de Munain,A., et al. (2013) The LRRK2 G2019S mutant exacerbates basal autophagy through activation of the MEK/ERK pathway. Cell. Mol. Life Sci. CMLS, 70, 121-136.

20. Manzoni,C., Mamais,A., Dihanich,S., Abeti,R., Soutar,M.P.M., Plun-Favreau,H., Giunti,P., Tooze,S.A., Bandopadhyay,R. and Lewis,P.A. (2013) Inhibition of LRRK2 kinase activity stimulates macroautophagy. Biochim. Biophys. Acta BBA - Mol. Cell Res., 1833, 2900-2910.

21. Mortiboys,H., Johansen,K.K., Aasly,J.O. and Bandmann,O. (2010) Mitochondrial impairment in patients with Parkinson disease with the G2019S mutation in LRRK2. Neurology, 75, 20172020 .

22. Papkovskaia,T.D., Chau,K.-Y., Inesta-Vaquera,F., Papkovsky,D.B., Healy,D.G., Nishio,K., Staddon,J., Duchen,M.R., Hardy,J., Schapira,A.H.V., et al. (2012) G2019S leucine-rich repeat kinase 2 causes uncoupling protein-mediated mitochondrial depolarization. Hum. Mol. Genet., 21, 4201-4213.

23. Su,Y.-C. and Qi,X. (2013) Inhibition of excessive mitochondrial fission reduced aberrant autophagy and neuronal damage caused by LRRK2 G2019S mutation. Hum. Mol. Genet., 22, $4545-4561$.

24. Cherra,S.J., Steer,E., Gusdon,A.M., Kiselyov,K. and Chu,C.T. (2013) Mutant LRRK2 Elicits Calcium Imbalance and Depletion of Dendritic Mitochondria in Neurons. Am. J. Pathol., 182, 474-484.

25. Wang, Yan,M.H., Fujioka,H., Liu,J., Wilson-Delfosse,A., Chen,S.G., Perry,G., Casadesus,G. and Zhu,X. (2012) LRRK2 regulates mitochondrial dynamics and function through direct interaction with DLP1. Hum. Mol. Genet., 21, 1931-1944.

26. Niu,J., Yu,M., Wang,C. and Xu,Z. (2012) Leucine-rich repeat kinase 2 disturbs mitochondrial dynamics via Dynamin-like protein. J. Neurochem., 122, 650-658.

27. Stafa,K., Tsika,E., Moser,R., Musso,A., Glauser,L., Jones,A., Biskup,S., Xiong,Y., Bandopadhyay,R., Dawson,V.L., et al. (2014) Functional interaction of Parkinson's diseaseassociated LRRK2 with members of the dynamin GTPase superfamily. Hum. Mol. Genet., 23, 2055-2077.

28. Perez Carrion,M., Pischedda,F., Biosa,A., Russo,I., Straniero,L., Civiero,L., Guida,M., Gloeckner,C.J., Ticozzi,N., Tiloca,C., et al. (2018) The LRRK2 Variant E193K Prevents 
Mitochondrial Fission Upon MPP+ Treatment by Altering LRRK2 Binding to DRP1. Front. Mol. Neurosci., 11, 64.

29. Twig,G., Elorza,A., Molina,A.J.A., Mohamed,H., Wikstrom,J.D., Walzer,G., Stiles,L., Haigh,S.E., Katz,S., Las,G., et al. (2008) Fission and selective fusion govern mitochondrial segregation and elimination by autophagy. EMBO J., 27, 433-446.

30. Narendra,D., Tanaka,A., Suen,D.-F. and Youle,R.J. (2008) Parkin is recruited selectively to impaired mitochondria and promotes their autophagy. J. Cell Biol., 183, 795-803.

31. Narendra,D.P., Jin,S.M., Tanaka,A., Suen,D.-F., Gautier,C.A., Shen,J., Cookson,M.R. and Youle,R.J. (2010) PINK1 is selectively stabilized on impaired mitochondria to activate Parkin. PLoS Biol., 8, e1000298.

32. Matsuda,N., Sato,S., Shiba,K., Okatsu,K., Saisho,K., Gautier,C.A., Sou,Y.-S., Saiki,S., Kawajiri,S., Sato,F., et al. (2010) PINK1 stabilized by mitochondrial depolarization recruits Parkin to damaged mitochondria and activates latent Parkin for mitophagy. J. Cell Biol., 189, 211-221.

33. Vives-Bauza,C., de Vries,R.L.A., Tocilescu,M. and Przedborski,S. (2010) PINK1/Parkin direct mitochondria to autophagy. Autophagy, 6, 315-316.

34. Pickrell,A.M. and Youle,R.J. (2015) The Roles of PINK1, Parkin, and Mitochondrial Fidelity in Parkinson's Disease. Neuron, 85, 257-273.

35. McWilliams,T.G. and Muqit,M.M. (2017) PINK1 and Parkin: emerging themes in mitochondrial homeostasis. Curr. Opin. Cell Biol., 45, 83-91.

36. Buhlman,L., Damiano,M., Bertolin,G., Ferrando-Miguel,R., Lombès,A., Brice,A. and Corti,O. (2014) Functional interplay between Parkin and Drp1 in mitochondrial fission and clearance. Biochim. Biophys. Acta BBA - Mol. Cell Res., 1843, 2012-2026.

37. Bertolin,G., Ferrando-Miguel,R., Jacoupy,M., Traver,S., Grenier,K., Greene,A.W., Dauphin,A., Waharte,F., Bayot,A., Salamero,J., et al. (2013) The TOMM machinery is a molecular switch in PINK1 and PARK2/PARKIN-dependent mitochondrial clearance. Autophagy, 9, 18011817.

38. Bertolin,G., Jacoupy,M., Traver,S., Ferrando-Miguel,R., Saint Georges,T., Grenier,K., ArdilaOsorio,H., Muriel,M.-P., Takahashi,H., Lees,A.J., et al. (2015) Parkin maintains mitochondrial levels of the protective Parkinson's disease-related enzyme 17- $\beta$ hydroxysteroid dehydrogenase type 10. Cell Death Differ., 22, 1563-1576.

39. Vives-Bauza,C., Zhou,C., Huang,Y., Cui,M., de Vries,R.L.A., Kim,J., May,J., Tocilescu,M.A., Liu,W., Ko,H.S., et al. (2010) PINK1-dependent recruitment of Parkin to mitochondria in mitophagy. Proc. Natl. Acad. Sci. U. S. A., 107, 378-383.

40. West,A.B., Moore,D.J., Choi,C., Andrabi,S.A., Li,X., Dikeman,D., Biskup,S., Zhang,Z., Lim,K.L., Dawson,V.L., et al. (2007) Parkinson's disease-associated mutations in LRRK2 link enhanced GTP-binding and kinase activities to neuronal toxicity. Hum. Mol. Genet., 16, 223232.

41. Webber,P.J., Smith,A.D., Sen,S., Renfrow,M.B., Mobley,J.A. and West,A.B. (2011) Autophosphorylation in the Leucine-Rich Repeat Kinase 2 (LRRK2) GTPase Domain Modifies Kinase and GTP-Binding Activities. J. Mol. Biol., 412, 94-110. 
42. Ito,G., Fujimoto,T., Kamikawaji,S., Kuwahara,T. and Iwatsubo,T. (2014) Lack of correlation between the kinase activity of LRRK2 harboring kinase-modifying mutations and its phosphorylation at Ser910, 935, and Ser955. PloS One, 9, e97988.

43. Deng,X., Dzamko,N., Prescott,A., Davies,P., Liu,Q., Yang,Q., Lee,J.-D., Patricelli,M.P., Nomanbhoy,T.K., Alessi,D.R., et al. (2011) Characterization of a selective inhibitor of the Parkinson's disease kinase LRRK2. Nat. Chem. Biol., 7, 203-205.

44. Tanaka,A., Cleland,M.M., Xu,S., Narendra,D.P., Suen,D.-F., Karbowski,M. and Youle,R.J. (2010) Proteasome and p97 mediate mitophagy and degradation of mitofusins induced by Parkin. $J$. Cell Biol., 191, 1367-1380.

45. Nivon,M., Richet,E., Codogno,P., Arrigo,A.-P. and Kretz-Remy,C. (2009) Autophagy activation by NFkappaB is essential for cell survival after heat shock. Autophagy, 5, 766-783.

46. Zhao,Y., Gong,S., Shunmei,E. and Zou,J. (2009) Induction of macroautophagy by heat. Mol. Biol. Rep., 36, 2323-2327.

47. Matheoud,D., Sugiura,A., Bellemare-Pelletier,A., Laplante,A., Rondeau,C., Chemali,M., Fazel,A., Bergeron,J.J., Trudeau,L.-E., Burelle,Y., et al. (2016) Parkinson's Disease-Related Proteins PINK1 and Parkin Repress Mitochondrial Antigen Presentation. Cell, 166, 314-327.

48. Rosado,C.J., Mijaljica,D., Hatzinisiriou,I., Prescott,M. and Devenish,R.J. (2008) Rosella: a fluorescent $\mathrm{pH}$-biosensor for reporting vacuolar turnover of cytosol and organelles in yeast. Autophagy, 4, 205-213.

49. Charmpilas,N., Kounakis,K. and Tavernarakis,N. (2017) Monitoring Mitophagy During Aging in Caenorhabditis elegans. Methods Mol. Biol. Clifton NJ, 10.1007/7651_2017_18.

50. Gautier,C.A., Erpapazoglou,Z., Mouton-Liger,F., Muriel,M.P., Cormier,F., Bigou,S., Duffaure,S., Girard,M., Foret,B., Iannielli,A., et al. (2016) The endoplasmic reticulum-mitochondria interface is perturbed in PARK2 knockout mice and patients with PARK2 mutations. Hum. Mol. Genet., 25, 2972-2984.

51. Cassidy-Stone,A., Chipuk,J.E., Ingerman,E., Song,C., Yoo,C., Kuwana,T., Kurth,M.J., Shaw,J.T., Hinshaw,J.E., Green,D.R., et al. (2008) Chemical inhibition of the mitochondrial division dynamin reveals its role in Bax/Bak-dependent mitochondrial outer membrane permeabilization. Dev. Cell, 14, 193-204.

52. Chang,C.-R. and Blackstone,C. (2010) Dynamic regulation of mitochondrial fission through modification of the dynamin-related protein Drp1. Ann. N. Y. Acad. Sci., 1201, 34-39.

53. Cereghetti,G.M., Stangherlin,A., Martins de Brito,O., Chang,C.R., Blackstone,C., Bernardi,P. and Scorrano,L. (2008) Dephosphorylation by calcineurin regulates translocation of Drp1 to mitochondria. Proc. Natl. Acad. Sci. U. S. A., 105, 15803-15808.

54. Singh,A., Zhi,L. and Zhang,H. (2018) LRRK2 and mitochondria: Recent advances and current views. Brain Res., 10.1016/j.brainres.2018.06.010.

55. Saez-Atienzar,S., Bonet-Ponce,L., Blesa,J.R., Romero,F.J., Murphy,M.P., Jordan,J. and Galindo,M.F. (2014) The LRRK2 inhibitor GSK2578215A induces protective autophagy in SH-SY5Y cells: involvement of Drp-1-mediated mitochondrial fission and mitochondrialderived ROS signaling. Cell Death Dis., 5, e1368. 
56. Yue,M., Hinkle,K.M., Davies,P., Trushina,E., Fiesel,F.C., Christenson,T.A., Schroeder,A.S., Zhang,L., Bowles,E., Behrouz,B., et al. (2015) Progressive dopaminergic alterations and mitochondrial abnormalities in LRRK2 G2019S knock-in mice. Neurobiol. Dis., 78, 172-195.

57. Schapansky,J., Nardozzi,J.D., Felizia,F. and LaVoie,M.J. (2014) Membrane recruitment of endogenous LRRK2 precedes its potent regulation of autophagy. Hum. Mol. Genet., 23, 42014214.

58. Gómez-Suaga,P., Luzón-Toro,B., Churamani,D., Zhang,L., Bloor-Young,D., Patel,S., Woodman,P.G., Churchill,G.C. and Hilfiker,S. (2012) Leucine-rich repeat kinase 2 regulates autophagy through a calcium-dependent pathway involving NAADP. Hum. Mol. Genet., 21, $511-525$.

59. Su,Y.-C., Guo,X. and Qi,X. (2015) Threonine 56 phosphorylation of Bcl-2 is required for LRRK2 G2019S-induced mitochondrial depolarization and autophagy. Biochim. Biophys. Acta, 1852, $12-21$.

60. Sánchez-Danés,A., Richaud-Patin,Y., Carballo-Carbajal,I., Jiménez-Delgado,S., Caig,C., Mora,S., Di Guglielmo,C., Ezquerra,M., Patel,B., Giralt,A., et al. (2012) Disease-specific phenotypes in dopamine neurons from human iPS-based models of genetic and sporadic Parkinson's disease. EMBO Mol. Med., 4, 380-395.

61. Alegre-Abarrategui,J., Christian,H., Lufino,M.M.P., Mutihac,R., Venda,L.L., Ansorge,O. and Wade-Martins,R. (2009) LRRK2 regulates autophagic activity and localizes to specific membrane microdomains in a novel human genomic reporter cellular model. Hum. Mol. Genet., 18, 4022-4034.

62. Orenstein,S.J., Kuo,S.-H., Tasset,I., Arias,E., Koga,H., Fernandez-Carasa,I., Cortes,E., Honig,L.S., Dauer,W., Consiglio,A., et al. (2013) Interplay of LRRK2 with chaperone-mediated autophagy. Nat. Neurosci., 16, 394-406.

63. Lee,J.J., Sanchez-Martinez,A., Zarate,A.M., Benincá,C., Mayor,U., Clague,M.J. and Whitworth,A.J. (2018) Basal mitophagy is widespread in Drosophila but minimally affected by loss of Pink1 or parkin. J. Cell Biol., 217, 1613-1622.

64. McWilliams,T.G., Prescott,A.R., Montava-Garriga,L., Ball,G., Singh,F., Barini,E., Muqit,M.M.K., Brooks,S.P. and Ganley,I.G. (2018) Basal Mitophagy Occurs Independently of PINK1 in Mouse Tissues of High Metabolic Demand. Cell Metab., 27, 439-449.e5.

65. Smith,G.A., Jansson,J., Rocha,E.M., Osborn,T., Hallett,P.J. and Isacson,O. (2016) Fibroblast Biomarkers of Sporadic Parkinson's Disease and LRRK2 Kinase Inhibition. Mol. Neurobiol., 53, 5161-5177.

66. Hsieh,C.-H., Shaltouki,A., Gonzalez,A.E., Bettencourt da Cruz,A., Burbulla,L.F., St. Lawrence,E., Schüle,B., Krainc,D., Palmer,T.D. and Wang,X. (2016) Functional Impairment in Miro Degradation and Mitophagy Is a Shared Feature in Familial and Sporadic Parkinson's Disease. Cell Stem Cell, 19, 709-724.

67. Gelmetti,V., De Rosa,P., Torosantucci,L., Marini,E.S., Romagnoli,A., Di Rienzo,M., Arena,G., Vignone,D., Fimia,G.M. and Valente,E.M. (2017) PINK1 and BECN1 relocalize at mitochondria-associated membranes during mitophagy and promote ER-mitochondria tethering and autophagosome formation. Autophagy, 13, 654-669. 
68. Celardo,I., Costa,A.C., Lehmann,S., Jones,C., Wood,N., Mencacci,N.E., Mallucci,G.R., Loh,S.H.Y. and Martins,L.M. (2016) Mitofusin-mediated ER stress triggers neurodegeneration in pink1/parkin models of Parkinson's disease. Cell Death Dis., 7, e2271.

69. McLelland,G.-L., Goiran,T., Yi,W., Dorval,G., Chen,C.X., Lauinger,N.D., Krahn,A.I., Valimehr,S., Rakovic,A., Rouiller,I., et al. (2018) Mfn2 ubiquitination by PINK1/parkin gates the p97-dependent release of ER from mitochondria to drive mitophagy. eLife, 7, e32866.

70. Friedman,J.R., Lackner,L.L., West,M., DiBenedetto,J.R., Nunnari,J. and Voeltz,G.K. (2011) ER tubules mark sites of mitochondrial division. Science, 334, 358-362.

71. Yang,J.-Y. and Yang,W.Y. (2013) Bit-by-bit autophagic removal of parkin-labelled mitochondria. Nat. Commun., 4, 2428.

72. de Brito,O.M. and Scorrano,L. (2008) Mitofusin 2 tethers endoplasmic reticulum to mitochondria. Nature, 456, 605-610.

73. Naon,D., Zaninello,M., Giacomello,M., Varanita,T., Grespi,F., Lakshminaranayan,S., Serafini,A., Semenzato,M., Herkenne,S., Hernández-Alvarez,M.I., et al. (2017) Reply to Filadi et al.: Does Mitofusin 2 tether or separate endoplasmic reticulum and mitochondria? Proc. Natl. Acad. Sci. U. S. A., 114, E2268-E2269.

74. Kornmann,B., Osman,C. and Walter,P. (2011) The conserved GTPase Gem1 regulates endoplasmic reticulum-mitochondria connections. Proc. Natl. Acad. Sci. U. S. A., 108, 1415114156.

75. Wong, Y.C., Ysselstein, D., Krainc, D. (2018) Mitochondria-lysosome contacts regulate mitochondrial fission via RAB7 GTP hydrolysis. Nature, 554, 382-386.

76. Yamano, K., Fogel A.I., Wang C., van der Bliek A.M., Youle R.J. (2014) Mitochondrial Rab GAPs govern autophagosome biogenesis during mitophagy. Elife, 3:e01612.

77. Eguchi, T., Kuwahara, T., Sakurai, M., Komori, T., Fujimoto, T., Ito, G., Yoshimura, S.I., Harada, A., Fukuda, M., Koike, M., Iwatsubo, T. (2018) LRRK2 and its substrate Rab GTPases are sequentially targeted onto stressed lysosomes and maintain their homeostasis. Proc. Natl. Acad. Sci. U. S. A., 115, E9115-E9124.

78. Di Maio, R., Hoffman, E.K., Rocha, E.M., Keeney, M.T., Sanders, L.H., De Miranda, B.R., Zharikov, A., Van Laar, A., Stepan, A.F., Lanz, T.A., et al. (2018) LRRK2 activation in idiopathic Parkinson's disease. Sci. Transl., 10, pii: eaar5429.

79. Periquet,M., Latouche,M., Lohmann,E., Rawal,N., De Michele,G., Ricard,S., Teive,H., Fraix,V., Vidailhet,M., Nicholl,D., et al. (2003) Parkin mutations are frequent in patients with isolated early-onset parkinsonism. Brain J. Neurol., 126, 1271-1278.

80. Kay,D.M., Bird,T.D., Zabetian,C.P., Factor,S.A., Samii,A., Higgins,D.S., Nutt,J., Roberts,J.W., Griffith,A., Leis,B.C., et al. (2006) Validity and utility of a LRRK2 G2019S mutation test for the diagnosis of Parkinson's disease. Genet. Test., 10, 221-227. 


\section{LEGENDS TO FIGURES}

\section{Figure 1: LRRK2 attenuates Parkin-dependent mitophagy}

(A) Representative images illustrating aggregation or clearance of the mitochondrial network (staining for the matrix protein, PMPCB) in cells overexpressing Parkin in the absence of LRRK2, and absence of mitochondrial clearance in cells expressing LRRK2 alone or together with Parkin, after treatment with CCCP $(48$ h, $10 \mu \mathrm{M})$. (B, C) Proportion of cells with aggregated mitochondrial staining (Aggregated) or without mitochondrial staining (No) in the conditions indicated. (D-F) Kinetics of mitochondrial aggregation (PMPCB staining) after CCCP exposure $(0 \mathrm{~h}-12 \mathrm{~h})(\mathrm{D})$, in the presence (dotted-line) or absence (full line) of LRRK2-IN-1 (E, F), in Parkin + LRRK2/LRRK2-G2019S conditions (E) or in Parkin-only or Parkin + LRRK2-D1994A conditions (F). Results are expressed as means $\pm \mathrm{SD}, n \geq 3$ independent experiments, with a minimum of 60-100 cells scored per condition in each experiment. Scale bar $=10 \mu \mathrm{m}$. ${ }^{*} p$-value $<0.05,{ }^{*} p$-value $<0.01,{ }^{* * *} p$-value $<0.001$ versus Parkin-only (B, C) or CCCP conditions (E). ${ }^{\# \#} p$-value $<0.001$ versus LRRK2 conditions.

Figure 2: LRRK2 kinase activity disturbs the protein-protein interactions induced by

\section{CCCP on mitochondria}

(A, C, E, G) Quantification of the efficiencies of FRET between TOMM70A as the acceptor and Parkin as the donor, or between Parkin as the acceptor and other subunits of the TOM complex (A, C), VDAC1 (A) or Drp1 (E) as donors; or between Mid51 (acceptor) and Drp1 (donor) $(\mathrm{G})$, in COS7 cells exposed to CCCP $(6 \mathrm{~h})$ expressing different LRRK2 variants, in the presence or absence of LRRK-IN-1 (B, D, F). Representative FRET images illustrating specific donor/acceptor pairs. The pseudocolor scale illustrates FRET efficiencies, reflecting individual pixel donor fluorescence changes after acceptor photobleaching. E: mean FRET 
efficiency within the region of interest (ROI, yellow frame). Scale bar $=2 \mu \mathrm{m}$. Results are expressed as means $\pm \mathrm{SD}, n \geq 3$ independent experiments, with a minimum of 7-12 cells analyzed per condition in each experiment. ${ }^{* * *} p<0.001$ versus Parkin-only conditions in the absence of LRRK2-IN-1, or ${ }^{\# \#} p$-value $<0.001$ versus corresponding LRRK2 or LRRK2G2019S conditions in the absence of LRRK2-IN-1.

\section{Figure 3: Thermal stress triggers mitophagy in human fibroblasts}

(A) Representative images illustrating the mtRosella signal in fibroblasts from a healthy subject in different thermal conditions: basal $\left(37^{\circ} \mathrm{C}\right)$, hyperthermic $\left(42^{\circ} \mathrm{C}\right)$ or hypothermic $\left(24^{\circ} \mathrm{C}\right)$. (B) Quantification of the ratio of the mean red-only fluorescence area to total red fluorescence per cell (\%) in the different temperature conditions. (C, D) Effect of treatment with the autophagy inhibitor 3-methyladenine (3-MA, 3 h) (C) or downregulation of the autophagy-related protein ATG5 (D, left panel) on the proportion of red-only fluorescence in control fibroblasts shifted to $24^{\circ} \mathrm{C}$. Right panel in (D): immunoblot showing the efficacy of ATG5 downregulation. Results are expressed as means $\pm \mathrm{SD}, n=3$ independent experiments, with a minimum of $12-20$ cells analyzed per condition in each experiment. ${ }^{* * *} p<0.001$ versus basal conditions $\left(37^{\circ} \mathrm{C}\right)(\mathrm{B})$, DMSO conditions (C) or control siRNA (D).

\section{Figure 4: The mitophagy triggered by thermal stress depends on PINK1 and Parkin}

(A, C) Representative images illustrating the mtRosella signal following a shift to $24^{\circ} \mathrm{C}$, in fibroblasts from a healthy subject transfected with a control siRNA or with PARK2- (Parkin) (A) or PINK1-specific (PINK1) (C) siRNAs. (B, D) Lower panels: quantification of the ratio of red-only fluorescence to total red fluorescence in control fibroblasts with and without Parkin silencing, after the cells were shifted to $24^{\circ} \mathrm{C}$. The dotted line indicates basal levels of 
mitophagy (cells maintained at $37^{\circ} \mathrm{C}$ ). Upper panels: immunoblots showing the efficacy of Parkin and PINK1 downregulation. Scale bar $=10 \mu \mathrm{m}$. Results are expressed as means $\pm \mathrm{SD}$, $n=3$ independent experiments, with a minimum of 12-20 cells analyzed per condition in each experiment. ${ }^{*} p<0.05$ and $* * p<0.01$ versus control siRNA $(\mathrm{G})$.

Figure 5: Parkin-dependent mitophagy is impaired in patients with $L R R K 2$ and $P A R K 2$ mutations.

(A) Relative abundance of red-only fluorescence in control fibroblasts $(n=4), L R R K 2$ G2019S fibroblasts $(n=4)$ and PARK2-fibroblasts $(n=4)$. (B) Relative abundance of redonly fluorescence in control fibroblasts $(n=3)$, LRRK2-G2019S fibroblasts $(n=3)$ and PARK2-fibroblasts $(n=3)$ in the presence or absence of LRRK2-IN-1. (C) Images illustrating the mtRosella signal in fibroblasts from a representative healthy subject, or representative patients with the $L R R K 2-\mathrm{G} 2019 \mathrm{~S}$ substitution (LRRK2-G2019S) or PARK2 mutations, in the presence or absence of LRRK2-IN-1 $(5 \mu \mathrm{M})$. The dotted line indicates the basal levels of mitophagy (cells maintained at $37^{\circ} \mathrm{C}$ ). Scale bar $10=\mu \mathrm{m}$. Results are expressed as means $\pm \mathrm{SD}$. Between 12 and 20 cells were analyzed per patient and condition. ${ }^{* *} p<0.01$ or ${ }^{* * *} p<0.001$ versus control (A). ${ }^{* *} p<0.01$ versus control in the absence of LRRK2-IN-1 treatment or ${ }^{\# \#} p$-value $<0.01$ versus LRRK2-G2019S in the absence of LRRK2IN-1 treatment (B).

Figure 6: The rescue of mitophagy induction in the presence of LRRK2-IN-1 is dependent on Drp1

(A) Images illustrating the mtRosella signal in fibroblasts from representative LRRK2 patients in the presence or absence of LRRK2-IN-1 $(5 \mu \mathrm{M}, 2 \mathrm{~h})$ in different transfection 
conditions: empty vector (pcDNA), or vector encoding functional Drp1 (Drp1) or dominantnegative Drp1 (Drp1-K38A). (B) Relative abundance of red-only fluorescence in the conditions shown in (A). (C) Representative images of mtRosella signals in fibroblasts from LRRK2 patients in the presence or absence of LRRK2-IN-1 or the Drp1 inhibitor Mdivi-1 (50 $\mu \mathrm{M}, 2$ h). (D) Relative abundance of red-only fluorescence in the conditions shown in (C). The dotted line indicates the basal levels of mitophagy (cells maintained at $37^{\circ} \mathrm{C}$ ). Scale bar $10=\mu \mathrm{m}$. Results are expressed as means $\pm \mathrm{SD}, n=3$ independent experiments, with a minimum of 12-20 cells analyzed per condition in each experiment. **p-value $<0.01$ or $* * * p$ $<0.001$ versus corresponding control conditions in the absence of LRRK2-IN-1. ${ }^{\# \#} p$-value $<$ 0.001 versus control conditions in the presence of LRRK2-IN-1. 


\section{TABLES}

Table 1. Age, sex and genotype of the donors of the skin fibroblasts used in this study

\begin{tabular}{|c|c|c|c|c|}
\hline Type & Age* & Sex & Gene & Protein \\
\hline Control 1 & 34 & $\mathrm{~F}$ & no mutation & normal \\
\hline Control 2 & 54 & M & no mutation & normal \\
\hline Control 3 & 53 & M & no mutation & normal \\
\hline Control 4 & 47 & M & no mutation & normal \\
\hline Control 5 & 66 & $\mathrm{~F}$ & no mutation & normal \\
\hline Patient $L R R K 21$ & 41 & $\mathrm{~F}$ & c. $6055 \mathrm{G}>\mathrm{A}$ & p.G2019S \\
\hline Patient $L R R K 22$ & 43 & $\mathrm{M}$ & c. $6055 \mathrm{G}>\mathrm{A}$ & p.G2019S \\
\hline Patient LRRK2 3 & 65 & $\mathrm{M}$ & c. $6055 \mathrm{G}>\mathrm{A}$ & p.G2019S \\
\hline Patient $L R R K 24$ & 63 & $\mathrm{~F}$ & c. $6055 \mathrm{G}>\mathrm{A}$ & p.G2019S \\
\hline Patient $L R R K 25$ & 70 & $\mathrm{~F}$ & c. $6055 \mathrm{G}>\mathrm{A}$ & p.G2019S \\
\hline Patient $L R R K 26$ & 76 & M & c. $6055 \mathrm{G}>\mathrm{A}$ & p.G2019S \\
\hline Patient PARK2 1 & 45 & $\mathrm{~F}$ & c.[(413-?)_(534+?)del];[155delA] & p.[A138GfsX7];[N52MfsX29] \\
\hline Patient PARK2 2 & 37 & $\mathrm{~F}$ & c. $\left[(172-?) \_(734+?) \mathrm{del}\right] ;\left[(172-?) \_(534+?) \mathrm{del}\right]$ & p.[N58EfsX29];[N58_Q178del] \\
\hline Patient PARK2 3 & 46 & $\mathrm{~F}$ & c. $\left[(872-?) \_(1083+?)\right.$ del $] ;[125 \mathrm{G}>\mathrm{C}]$ & p. $[\mathrm{A} 291 \mathrm{Vfs} X 8] ;[\mathrm{R} 42 \overline{\mathrm{P}}]$ \\
\hline Patient PARK2 4 & 51 & $\mathrm{~F}$ & c. $\left[(872-?) \_(1083+?) \mathrm{del}\right] ;[125 \mathrm{G}>\mathrm{C}]$ & p.[A291VfsX8];[R42P] \\
\hline Patient $P A R K 25$ & 50 & $\mathrm{M}$ & c. $[(535-?)(618+?) \mathrm{del}] ;[1321 \mathrm{~T}>\mathrm{C}]$ & p.[G179_A206del];[C441R] \\
\hline
\end{tabular}

*Age at biopsy (in years)

Table 2. Antibodies used for western blotting

\begin{tabular}{|c|c|c|c|c|}
\hline $\begin{array}{l}\text { Primary antibodies } \\
\text { Type }\end{array}$ & Company & Catalog \# & Host & Dilution \\
\hline PARK2, clone Park8 & Santa Cruz & sc-32282 & mouse & $1: 1000$ \\
\hline LRRK2 & Novus Biologicals & NB300-268 & rabbit & $1: 500$ \\
\hline PINK1 & Novus Biologicals & NB110-53818 & rabbit & $1: 1000$ \\
\hline Phospho-Drp1 (Ser616) & Cell Signaling & FF3455 & rabbit & $1: 1000$ \\
\hline Phospho-Drp1 (Ser637) & Cell Signaling & H4867 & rabbit & $1: 500$ \\
\hline Drp1 & Abcam & ab56788 & mouse & $1: 500$ \\
\hline Mfn2 & Abcam & ab56889 & mouse & $1: 1000$ \\
\hline Tubulin & Abcam & ab7991 & mouse & $1: 2000$ \\
\hline Actin & Abcam & ab3280 & mouse & $1: 4000$ \\
\hline $\begin{array}{l}\text { Secondary antibodies } \\
\text { Type }\end{array}$ & Company & & Host & Dilution \\
\hline $\begin{array}{l}\text { Horseradish peroxidase-conjugated } \\
\text { anti-mouse / anti-rabbit }\end{array}$ & $\begin{array}{l}\text { Jackson } \\
\text { ImmunoResearch }\end{array}$ & $\begin{array}{l}115-035-146 / \\
111-035-144\end{array}$ & goat & $1: 50000$ \\
\hline
\end{tabular}


Table 3. Antibodies used for immunocytochemical staining and FRET experiments

\begin{tabular}{lllll}
\hline $\begin{array}{l}\text { Primary antibodies } \\
\text { Type }\end{array}$ & Company & Catalog \# & Host & Dilution \\
\hline PARK2, clone Park8 & Millipore & MAB5512 & mouse & $1: 1000$ \\
PARK2 & Millipore & MAB5112 & rabbit & $1: 4000$ \\
TOMM20 & Santa Cruz & Sc-11415 & rabbit & $1: 1000$ \\
TOMM22 & Sigma & HPA003037 & rabbit & $1: 1000$ \\
TOMM40 & Santa Cruz & sc-11414 & rabbit & $1: 100$ \\
TOMM70A & Abcam & ab106193 & mouse & $1: 2000$ \\
VDAC1 & Abcam & ab15895 & rabbit & $1: 500$ \\
Drp1 & BD Biosciences & 611738 & mouse & $1: 2000$ \\
MiD51 & Santa Cruz & sc-86875 & rabbit & $1: 500$ \\
GFP & Abcam & ab13970 & chicken & $1: 4000$ \\
PMPCB & Proteintech & $16064-1-A P$ & rabbit & $1: 500$ \\
\hline Secondary antibodies & & & & \\
Type & Company & & Host & Dilution \\
\hline Alexa Fluor 405 anti-chicken & Abcam & ab175674 & goat & $1: 4000^{*}$ \\
Alexa Fluor 488 anti-mouse/rabbit & Invitrogen & A11070/A11029 & goat & $1: 4000 ; 1: 10000^{*}$ \\
Alexa Fluor 568 anti-mouse/rabbit & Invitrogen & A11036/A11031 & goat & $1: 10000^{*}$ \\
CY3-conjugated anti-rabbit & Sigma & C2306 & sheep & $1: 4000$ \\
\hline *Dilution used for FRET experiments & & & & \\
\hline
\end{tabular}




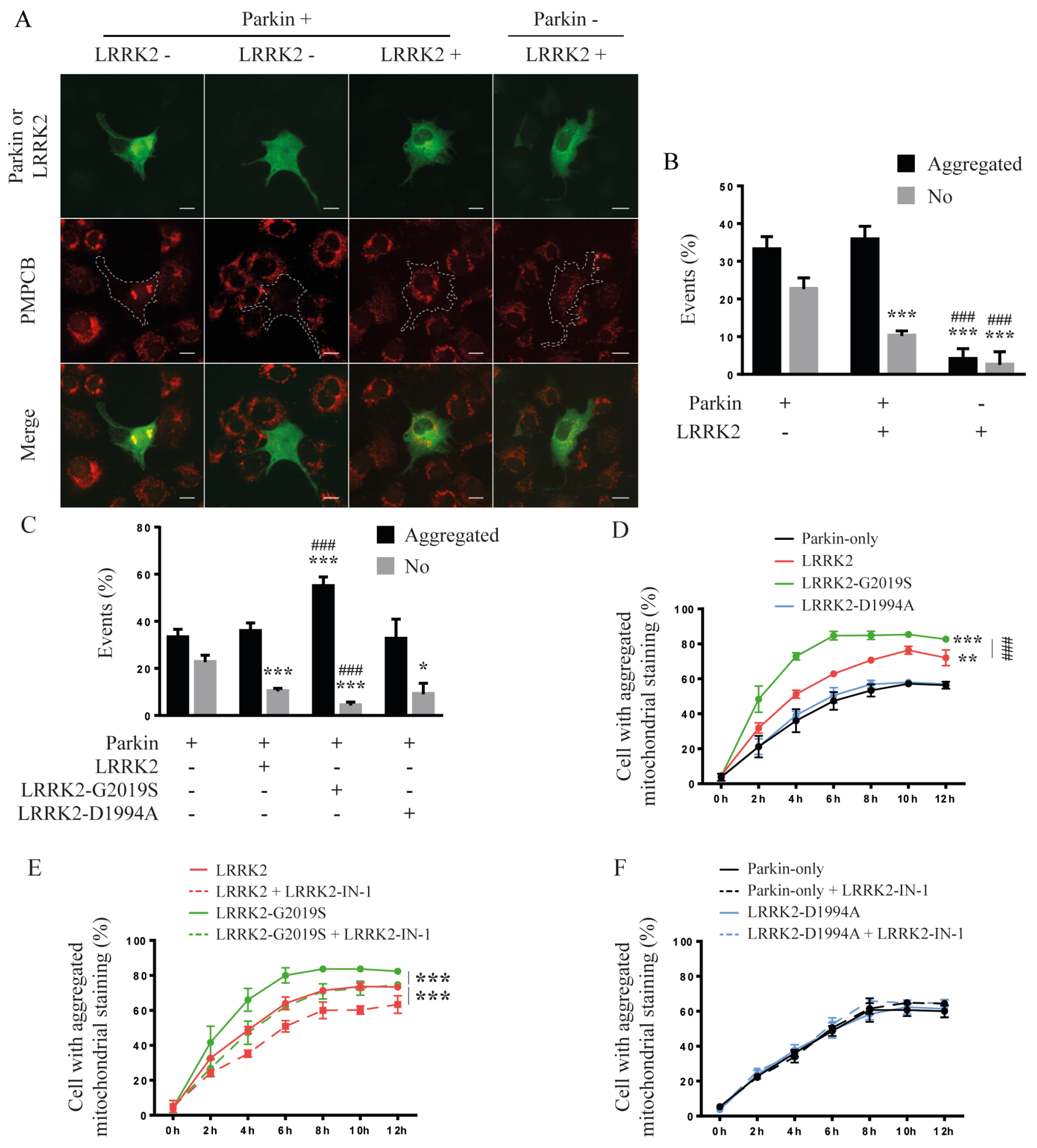


A

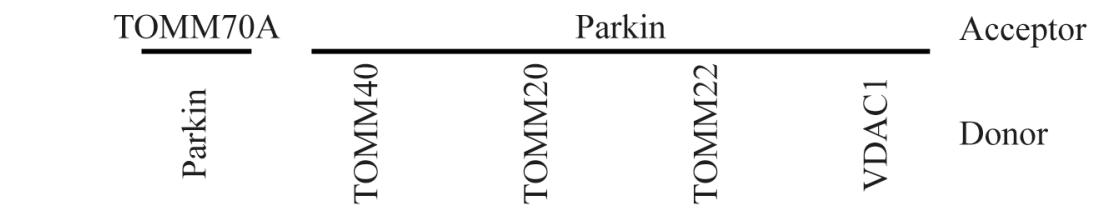

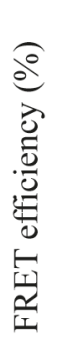

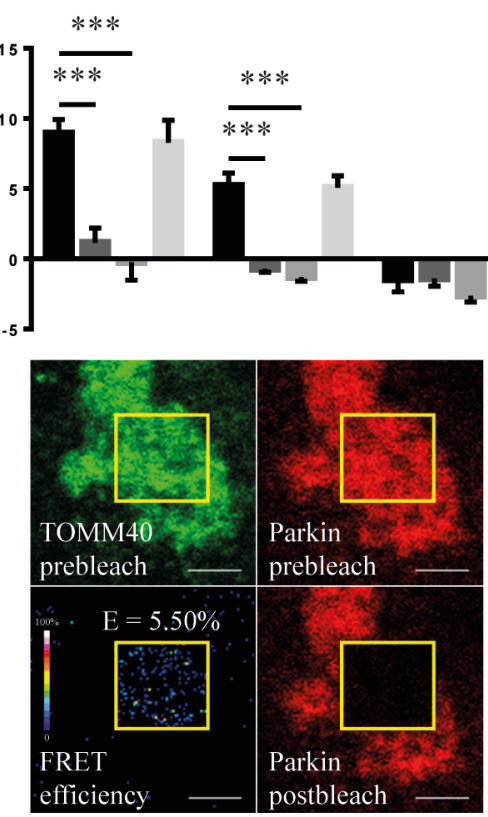

D

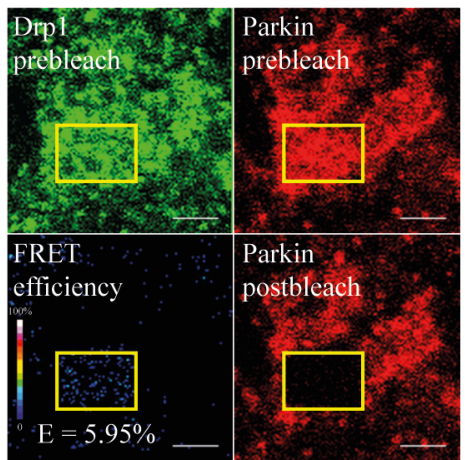

F

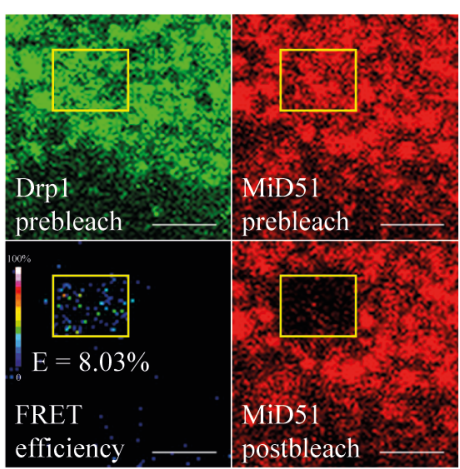

C

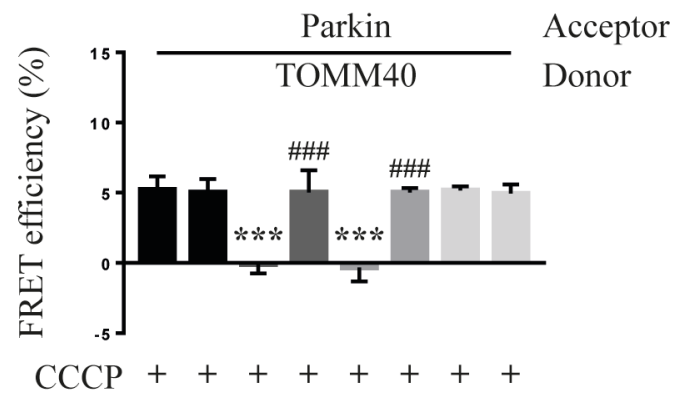

LRRK2-IN-1 - + - + - + +

E

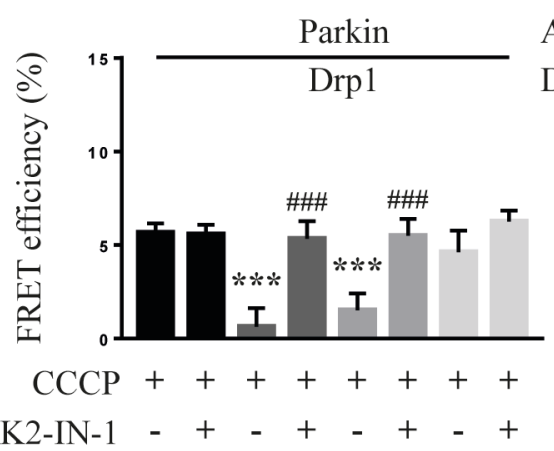

G




A

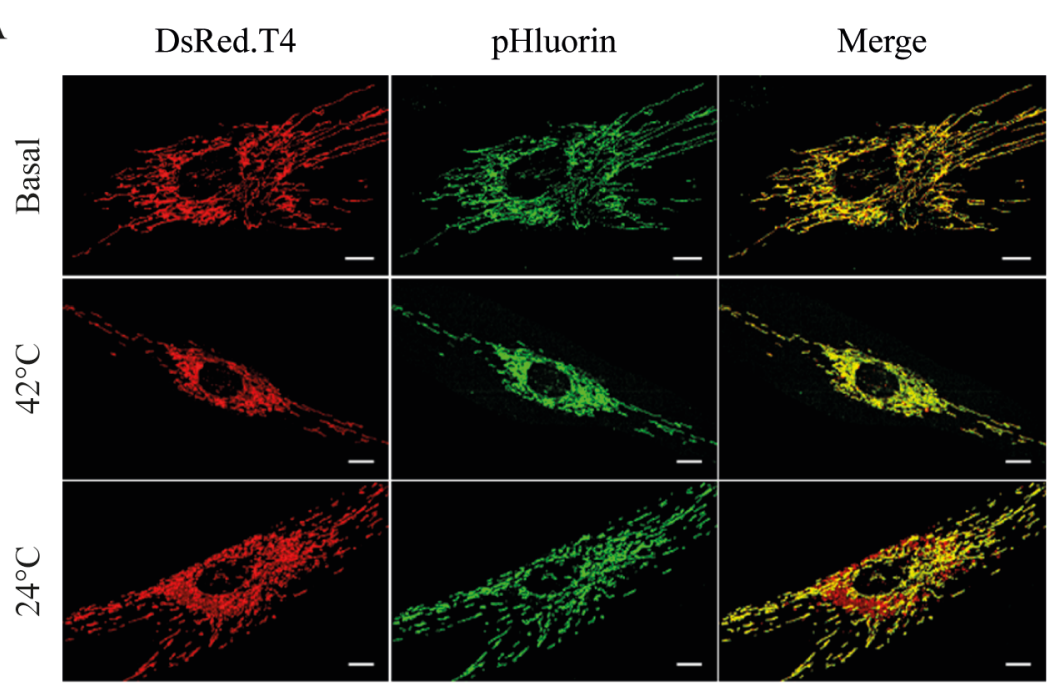

B
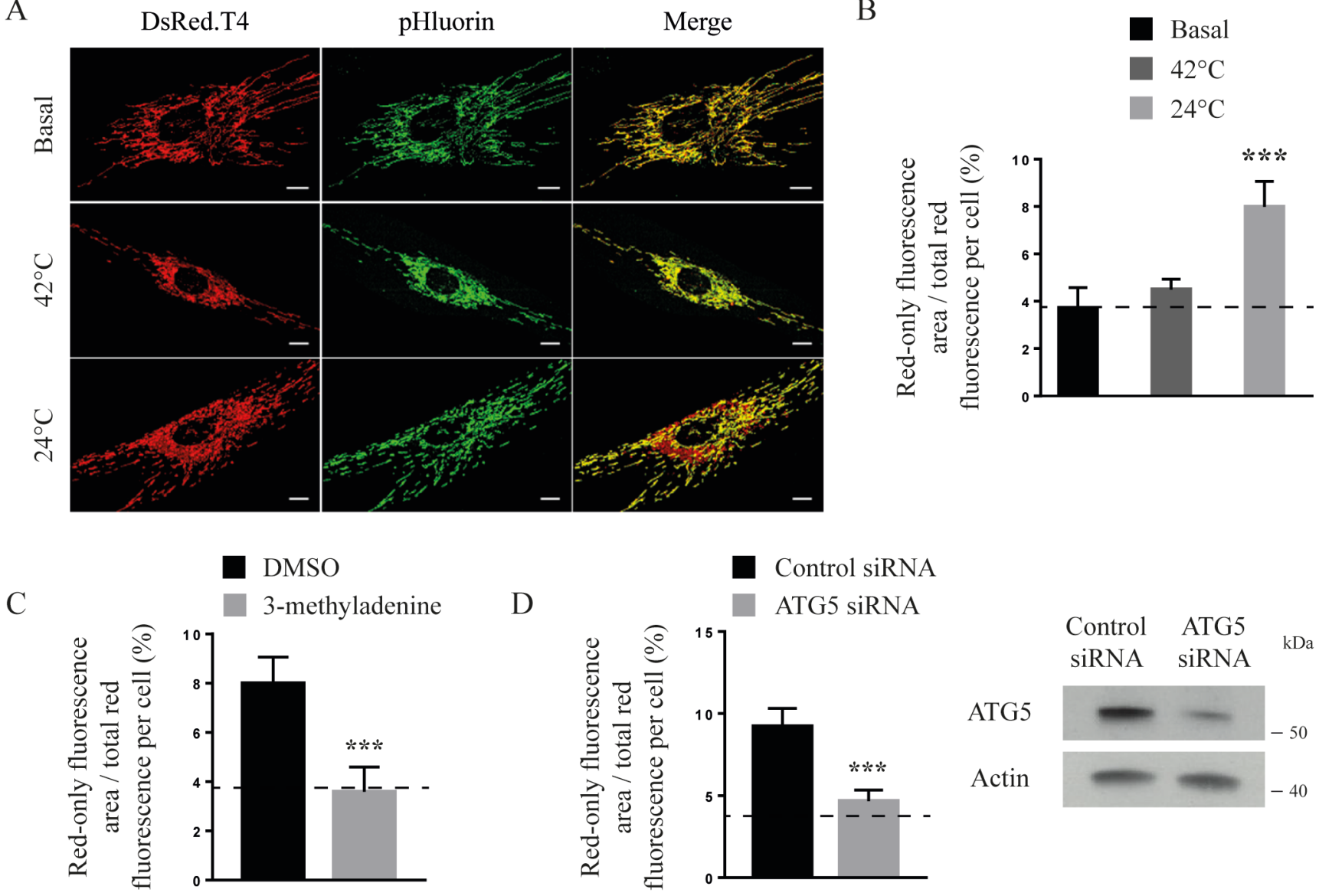

D
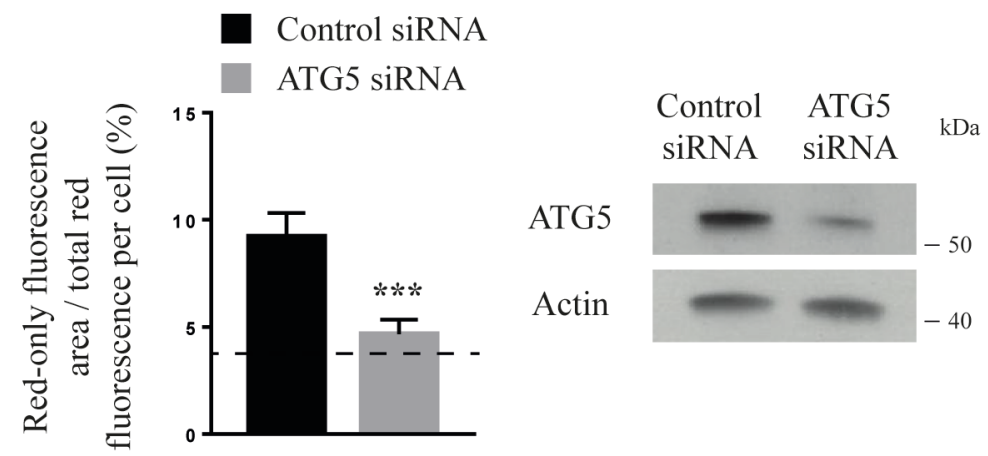

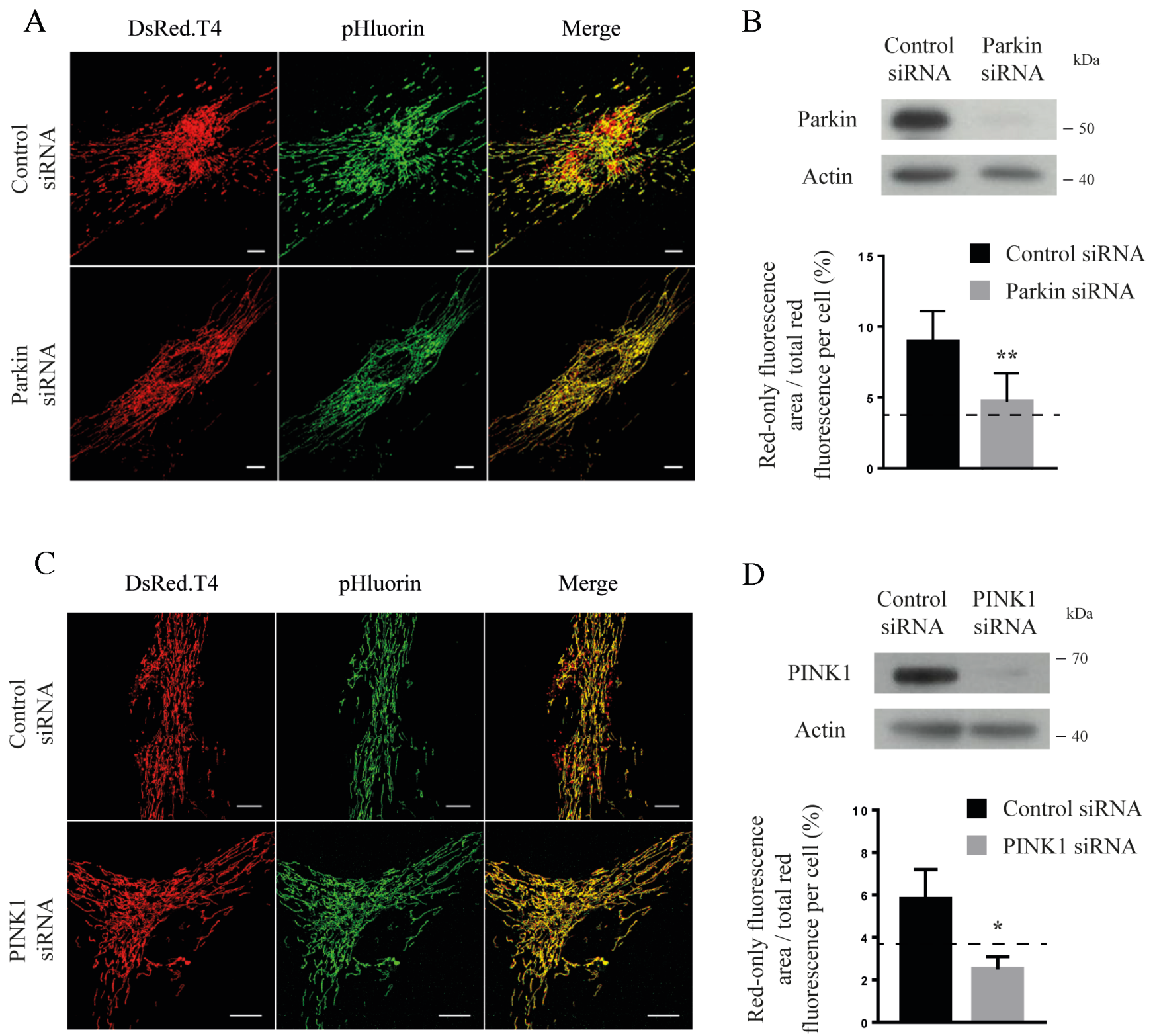

D
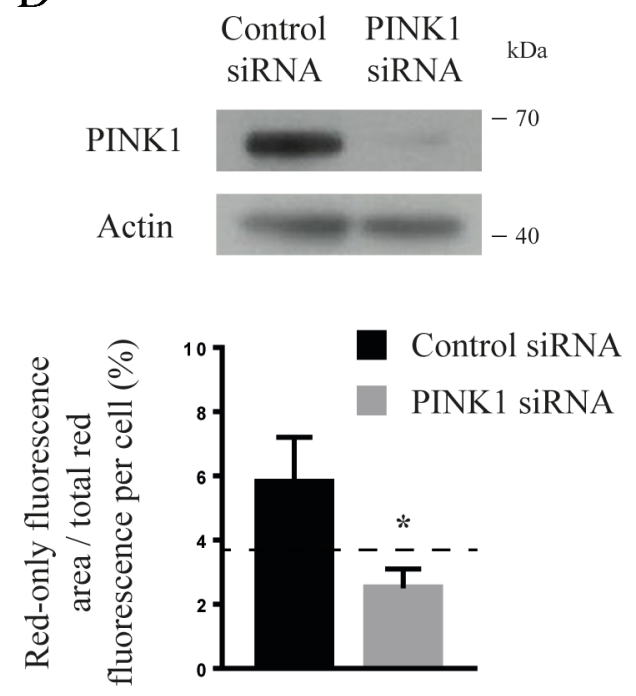
A

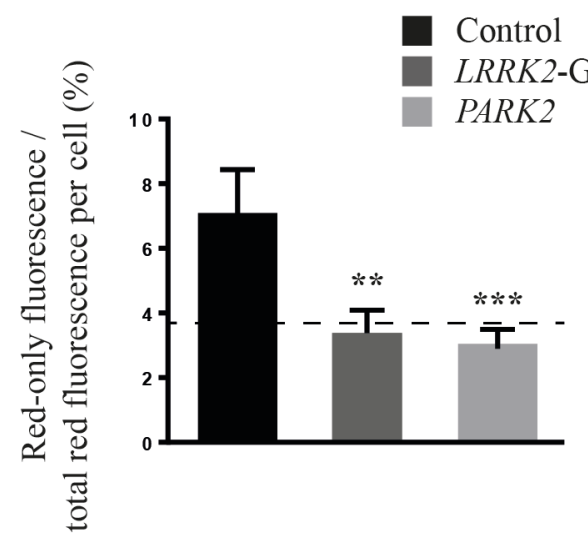

B

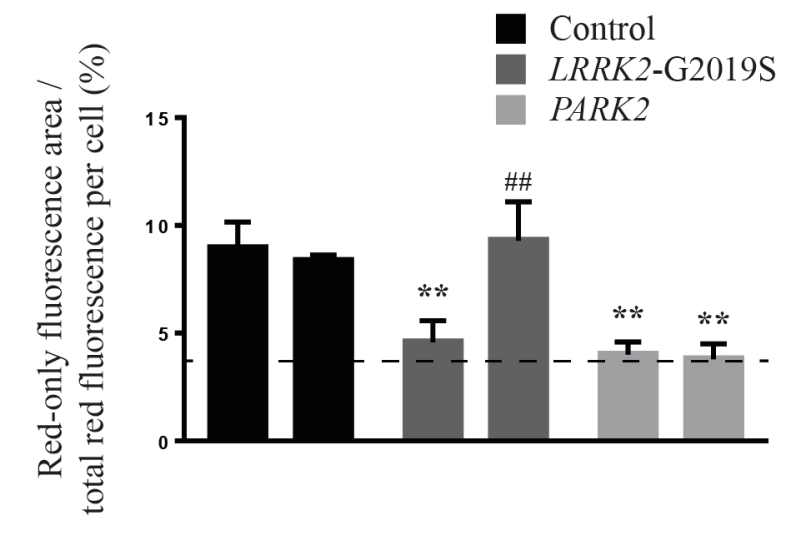

LRRK2-IN-1 - $\quad+\quad+\quad+\quad+\quad-\quad+$

C

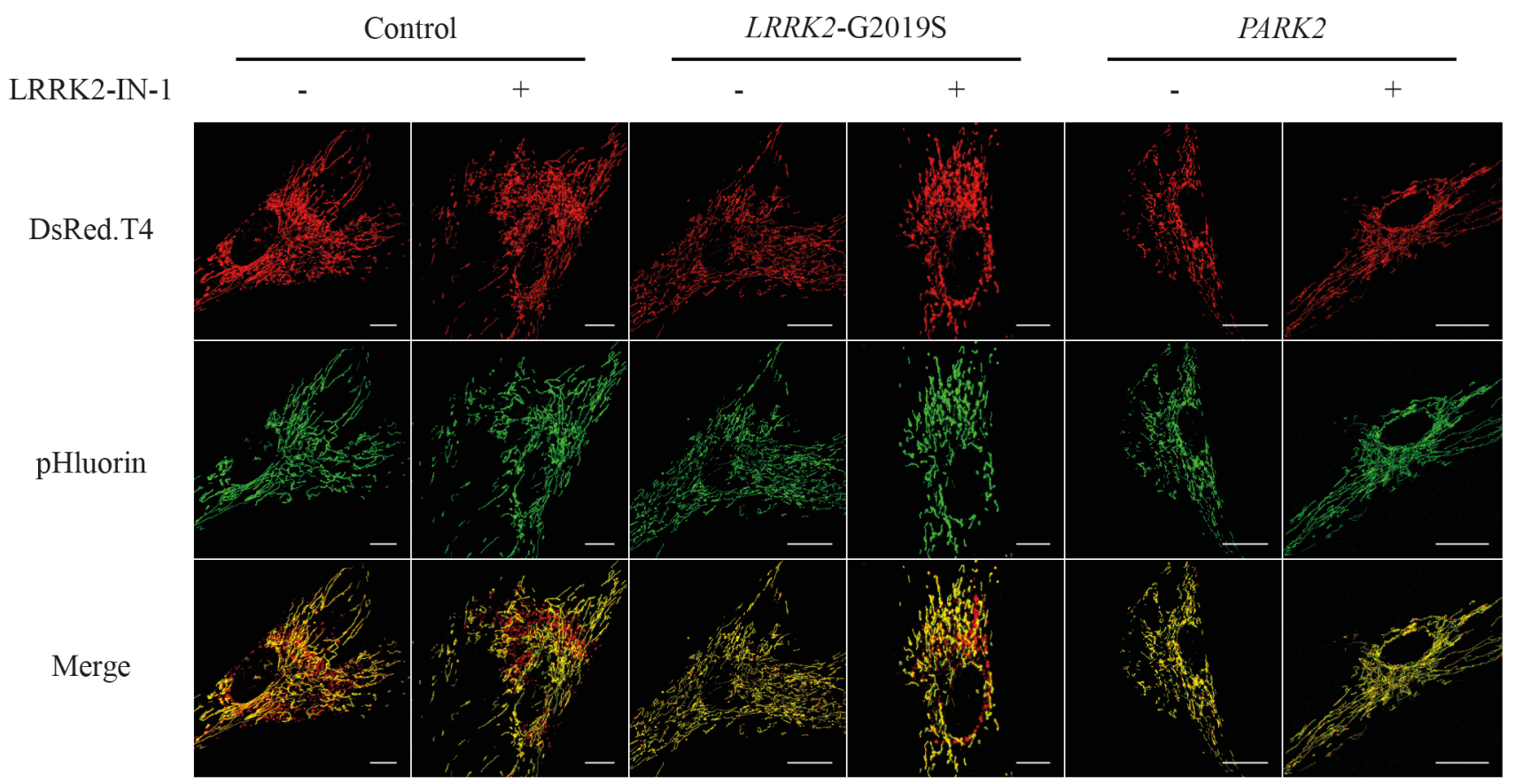




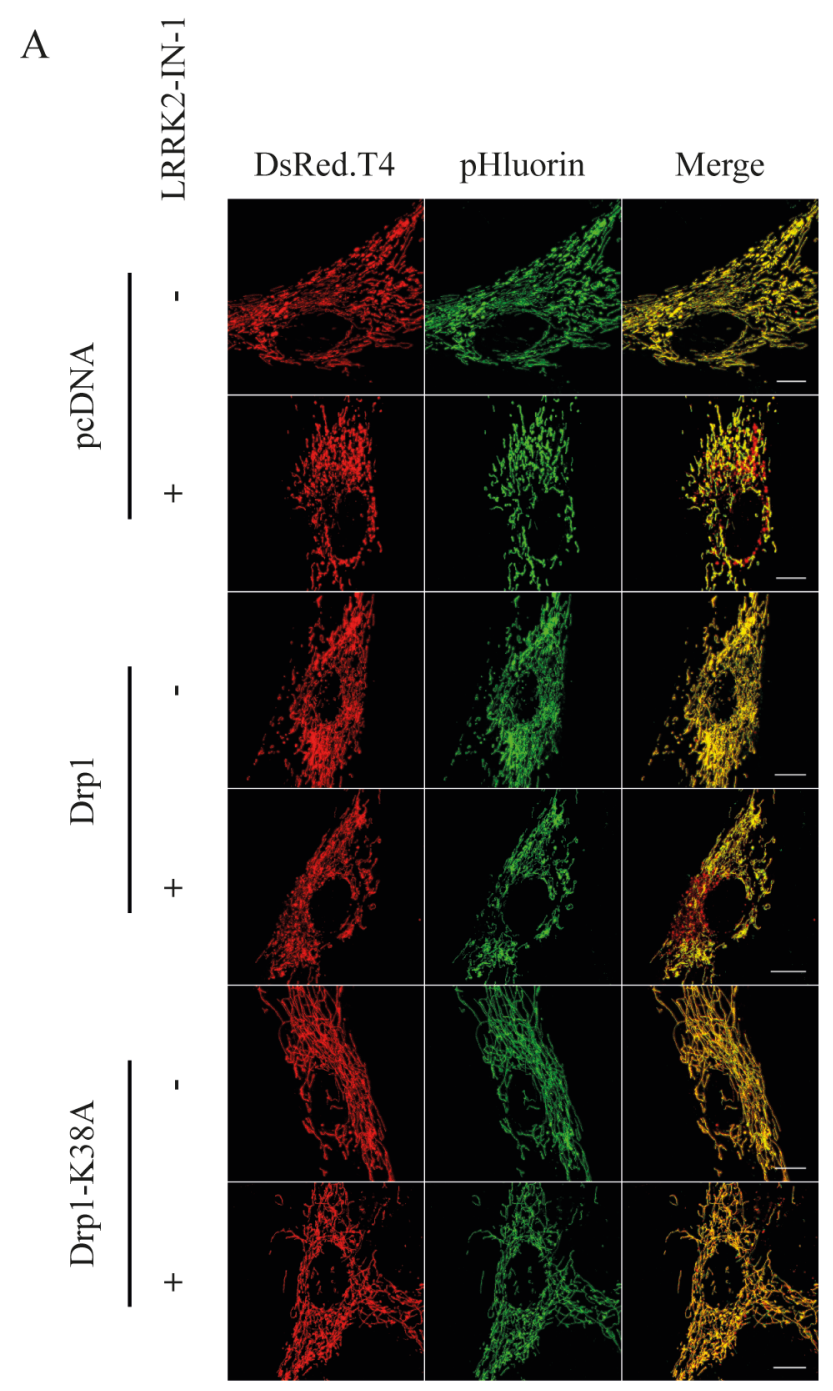

C

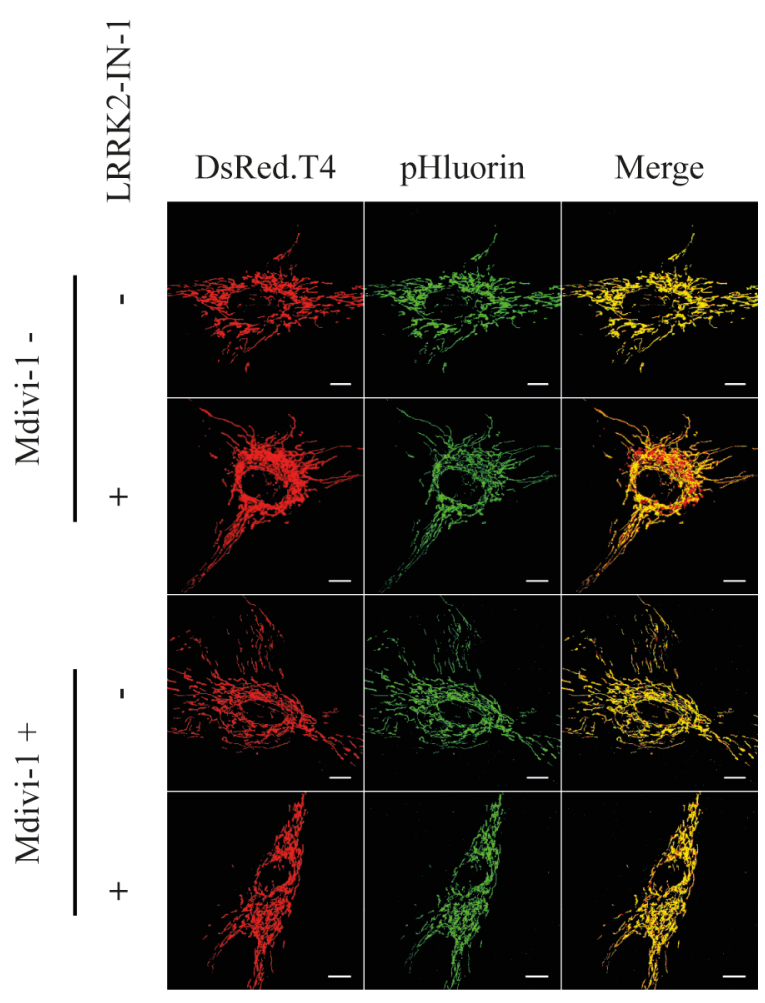

B

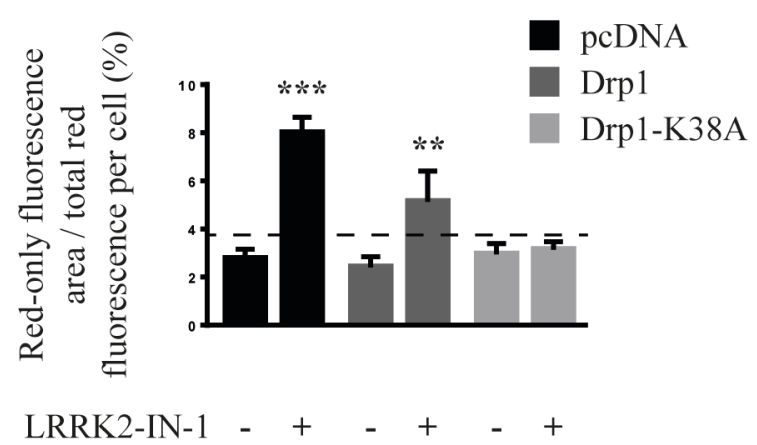

D

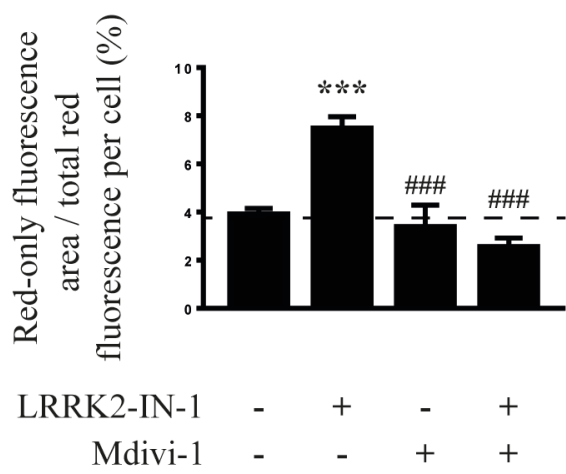




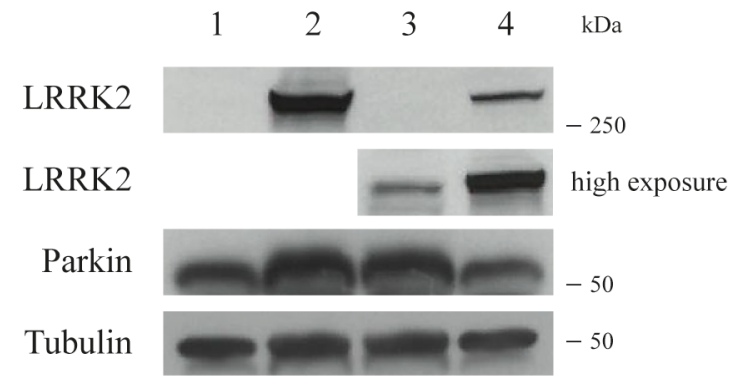


A

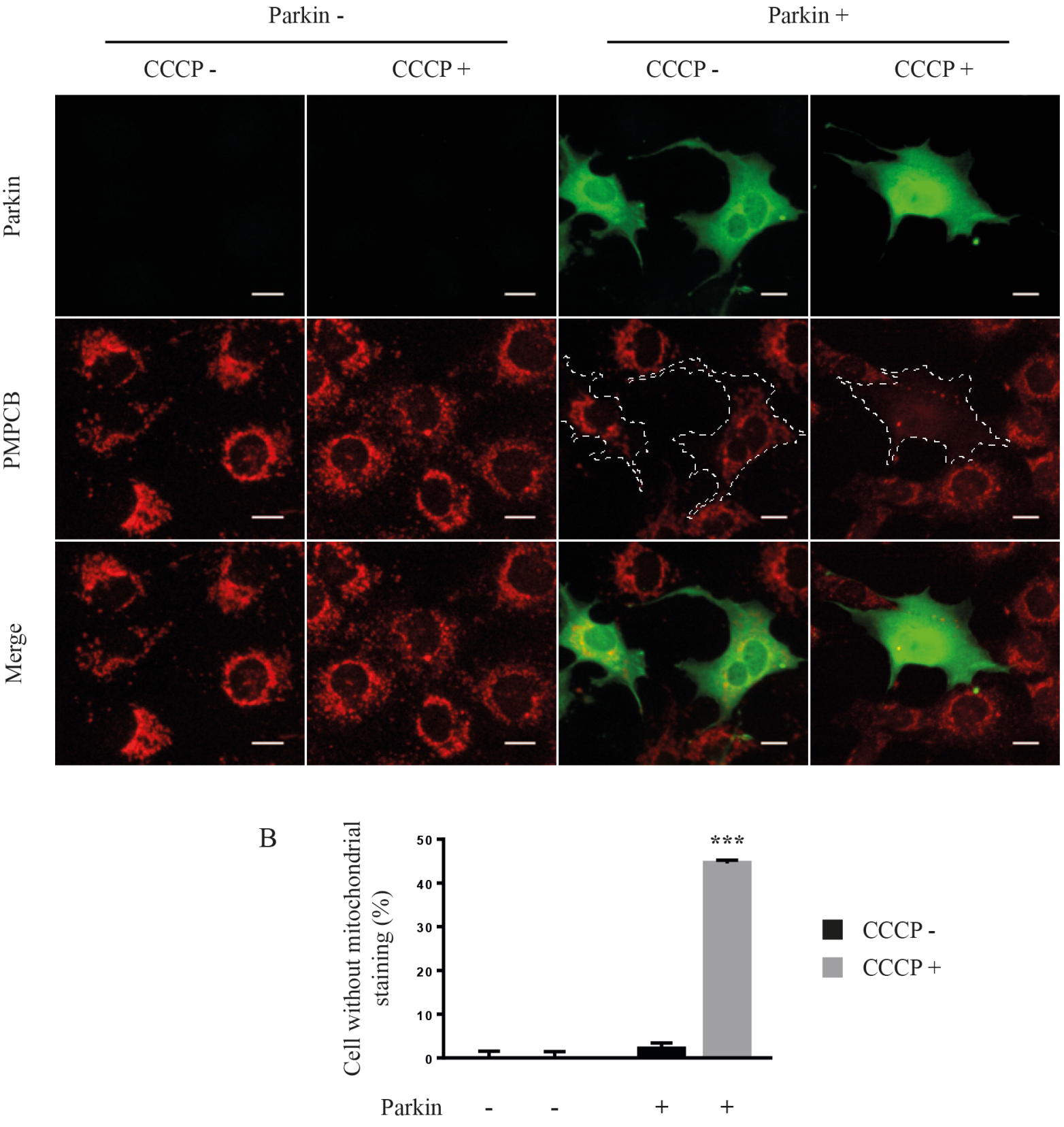




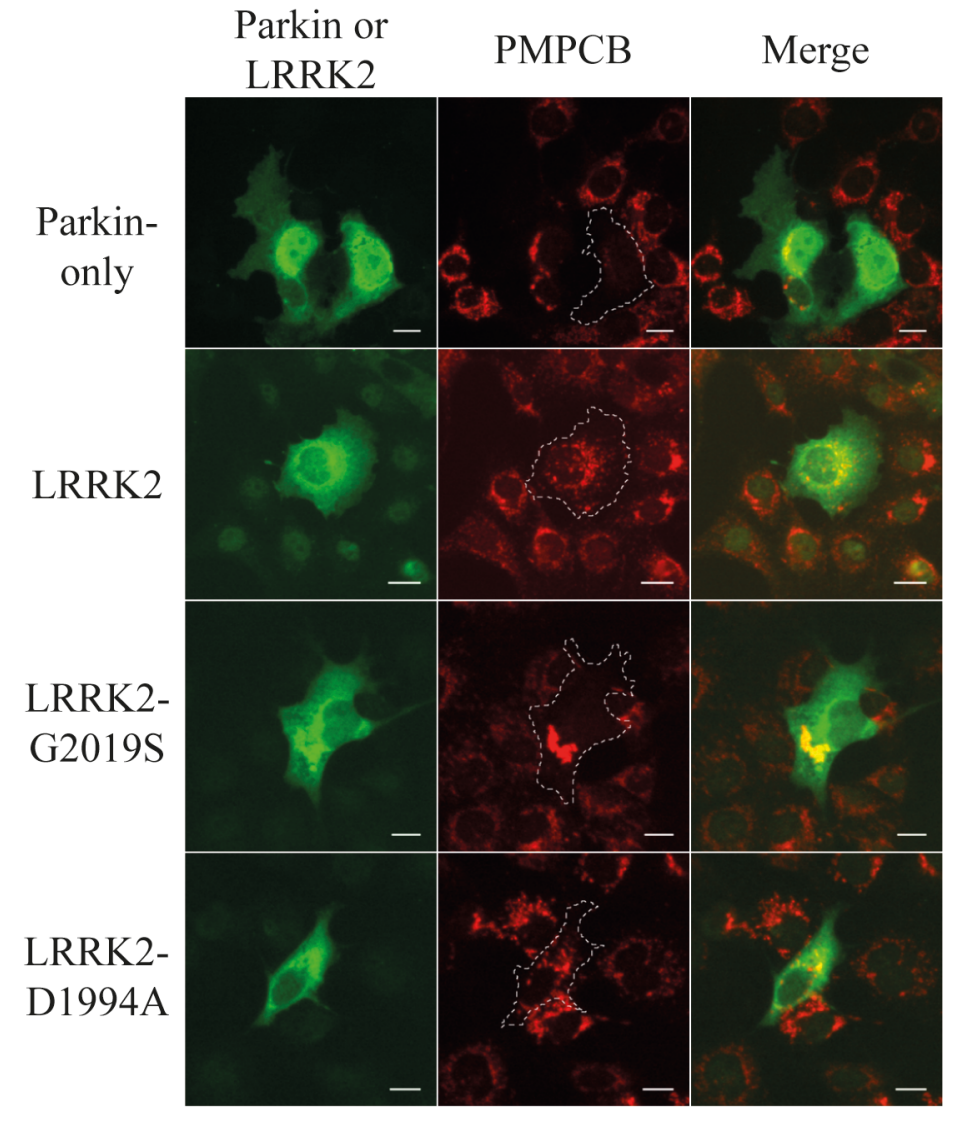


A

C
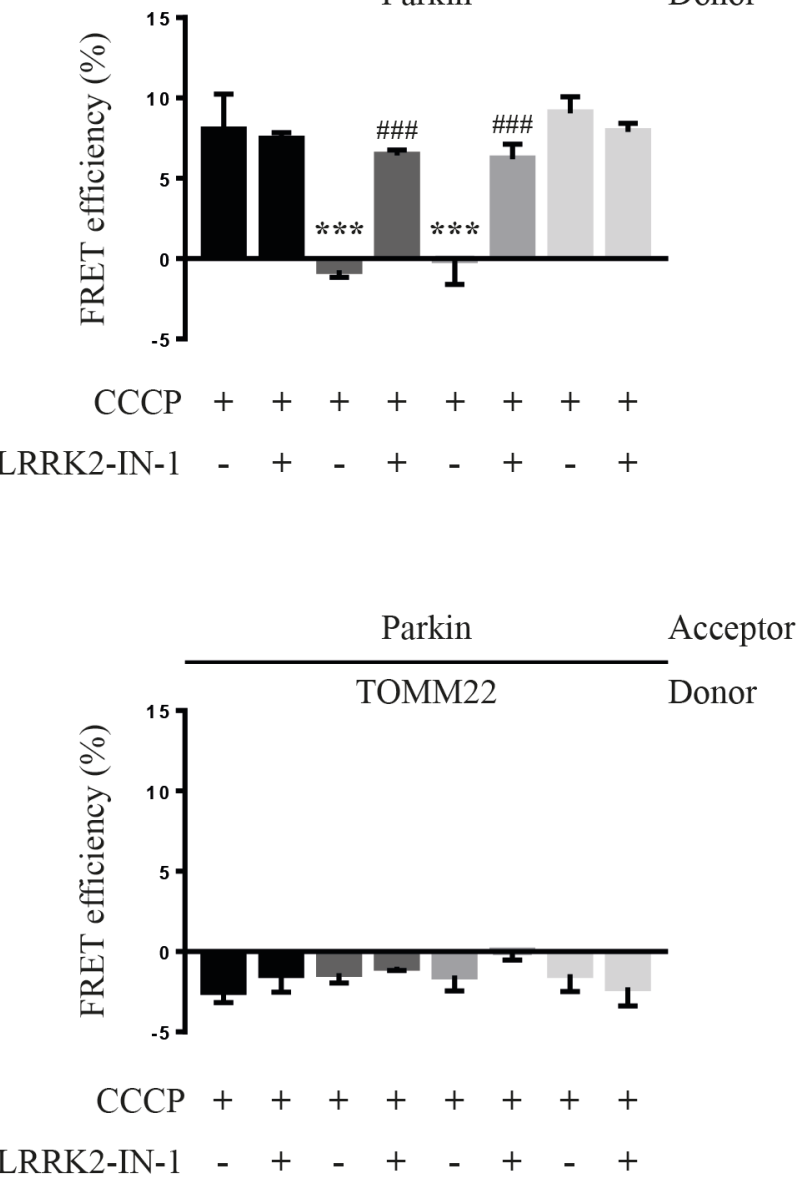

$$
\mathrm{CCCP}++++++++
$$

LRRK2-IN-1 - + - + - + - +

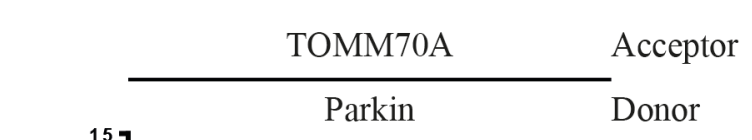

B
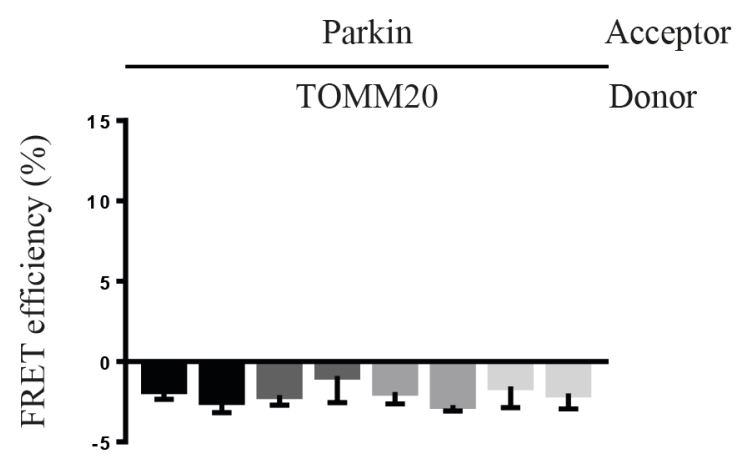

D

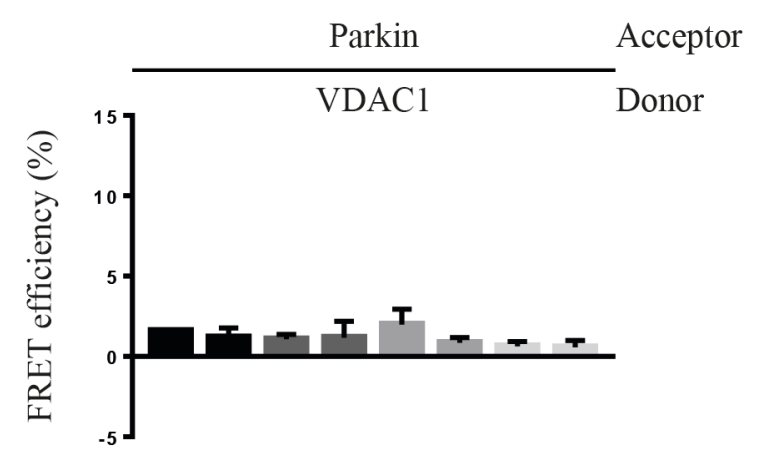

$\mathrm{CCCP}++++++++$

LRRK2-IN-1 - + - + - + - + 
A
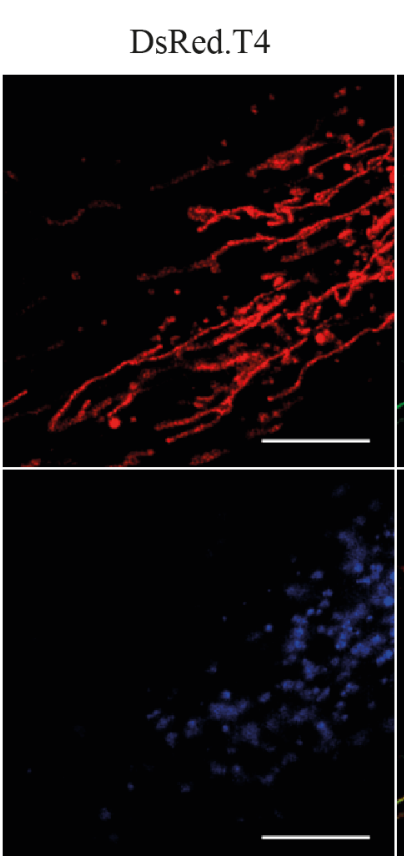

LysoTracker
pHluorin

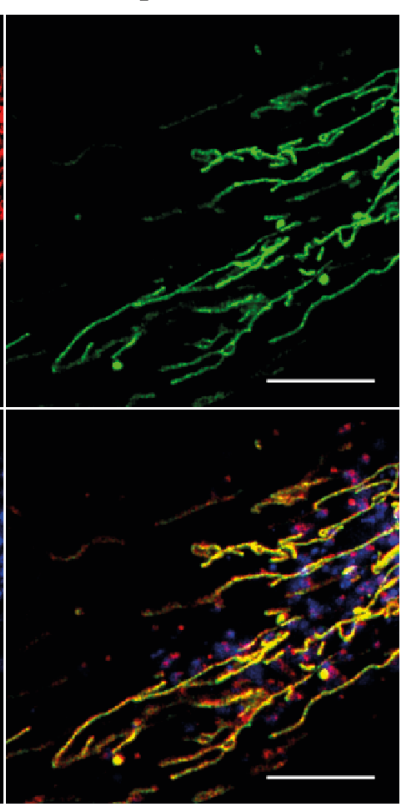

Merge

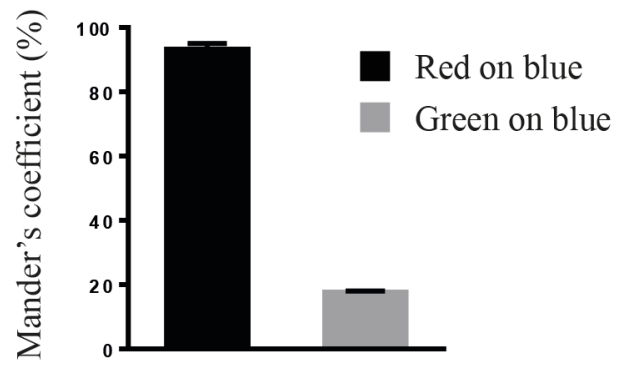


LRRK2

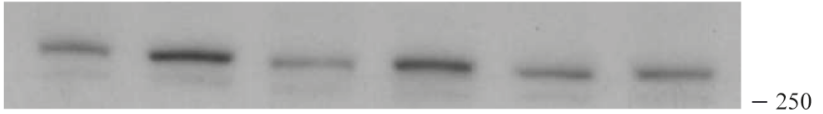

Actin

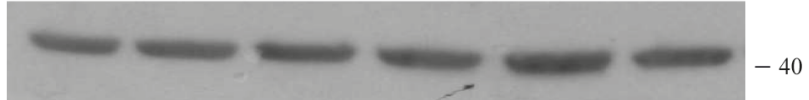


A

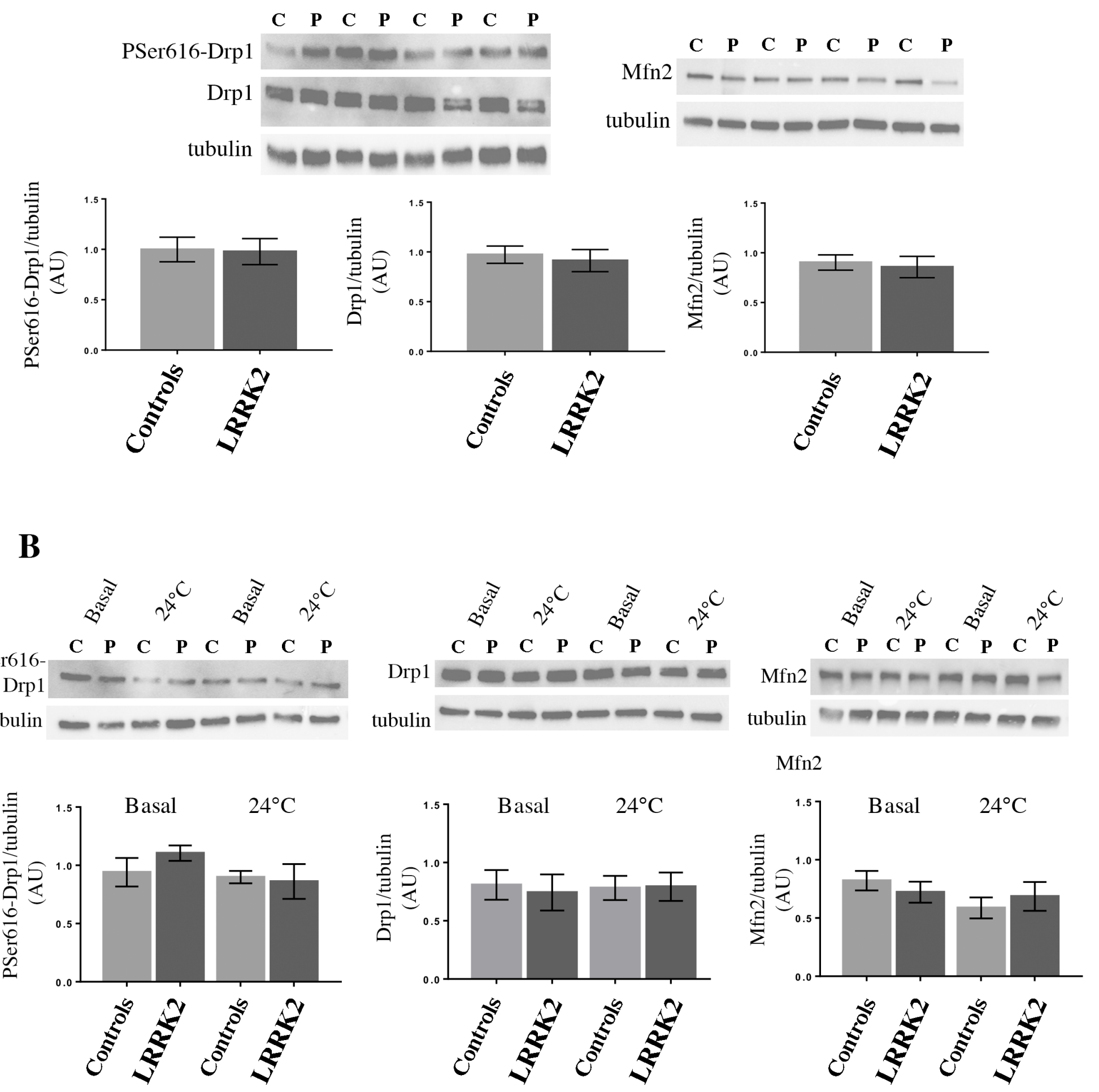


Figure S1: Levels of expression for LRRK2 variants and Parkin in COS7 cells

Western blot evaluating relative expression levels for exogenous normal LRRK2 (2), LRRK2G2019S (3) or LRRK2-D1994A (4) and exogenous Parkin (1-4) in transiently transfected COS7 cells. In (1) cells were transfected with the Parkin vector only.

\section{Figure S2: Model of Parkin-dependent mitophagy}

(A) Representative images illustrating the loss of the mitochondrial network (staining for mitochondrial matrix protein PMPCB) in COS7 cells transfected with empty vector (Parkin -) or with Parkin vector (Parkin + ), with and without CCCP treatment (48 h, $10 \mu \mathrm{M})$. (B) Proportion of cells without mitochondrial staining in the conditions shown in (A). Results are expressed as means $\pm \mathrm{SD}, n=3$ independent experiments, with a minimum of 100 cells per condition in each experiment. Scale bar $=10 \mu \mathrm{m} .{ }^{* * *} p$-value $<0.001$ versus Parkin + , CCCP

\section{Figure S3: Impact of LRRK2 variants on PINK1/Parkin-dependent mitophagy}

Representative images illustrating mitochondrial clearance or lack thereof (staining for the mitochondrial matrix protein, PMPCB) in cells expressing Parkin alone (Parkin-only) or with the indicated LRRK2 variants, after treatment with CCCP $(48 \mathrm{~h}, 10 \mu \mathrm{M})$. Note the aggregated mitochondrial network in cells expressing LRRK2-G2019S.

Figure S4: LRRK2 kinase activity disturbs the protein-protein interactions induced by CCCP on mitochondria

(A-D) Quantification of the efficiency of FRET between the indicated donor/acceptor pairs in different conditions (Parkin-only (black), LRRK2 (dark gray), LRRK2-G2019S (gray) and 
LRRK2-D1994A (light gray)) in COS7 cells treated with CCCP $(10 \mu \mathrm{M})$, with or without LRRK2-in-1 $(5 \mu \mathrm{M})$ for $6 \mathrm{~h}$. Results are expressed as means $\pm \mathrm{SD}, n \geq 3$ independent experiments, with a minimum of 7-12 cells analyzed per condition in each experiment. ${ }^{* * *} p<$

0.001 versus Parkin-only condition in the absence of LRRK2-IN-1, or ${ }^{\# \#} p$-value $<0.001$ versus corresponding LRRK2 or LRRK2-G2019S conditions, in the absence of LRRK2-IN-1.

\section{Figure S5: Red-only fluorescent vesicles colocalize with lysosomes}

(A) Images illustrating the mtRosella signal in a representative fibroblast from a healthy subject after a shift to $24^{\circ} \mathrm{C}$ and staining with the lysosomal dye, LysoTracker blue, and (B) index of colocalization between red-only fluorescence (red on blue) or green fluorescence (green on blue) and lysosomal staining (Mander's coefficient). Scale bar $5=\mu \mathrm{m}$.

Figure S6: LRRK2 detection in human fibroblasts from control subjects and PD patients

Western blot revealing the presence of LRRK2 in fibroblasts from representative control subjects and patients with $L R R K 2-\mathrm{G} 2019 \mathrm{~S}$ or PARK2 mutations.

Figure S7: Intracellular levels of Drp1, phosphorylated Drp1 and Mfn2 are similar in fibroblasts from control subjects and PD patients carrying the G2019S $L R R K 2$ mutation

Representative immunoblots illustrating a western blot analysis of whole cell lysates from patient- and control subject-derived fibroblasts, using antibodies against Drp1 phosphorylated on serine 616 (PSer616-Drp1), total Drp1 or Mfn2, under basal conditions (A, $n=4$ patients and $n=6$ controls) or following shift of the cells to $42^{\circ}$ ( $\mathrm{B}, n=2$ patients and $n=2$ controls). 
Table S1. PINK1/Parkin-dependent mitophagy in fibroblasts from PD patients

\begin{tabular}{|c|c|c|}
\hline Experiment & Subject & Mitophagy index \\
\hline \multirow{4}{*}{1} & Control 1 & 9.04 \\
\cline { 2 - 3 } & LRRK2 1 & 3.40 \\
\cline { 2 - 3 } & PARK2 4 & 3.68 \\
\hline \multirow{4}{*}{2} & Control 3 & 6.68 \\
\cline { 2 - 3 } & LRRK2 3 & 2.36 \\
\cline { 2 - 3 } & PARK2 1 & 2.68 \\
\hline \multirow{4}{*}{3} & Control 4 & 5.48 \\
\cline { 2 - 3 } & LRRK2 4 & 3.90 \\
\cline { 2 - 3 } & PARK2 5 & 2.18 \\
\hline \multirow{4}{*}{4} & Control 2 & 6.56 \\
\cline { 2 - 3 } & LRRK2 2 & 3.84 \\
\cline { 2 - 3 } & PARK2 2 & 3.13 \\
\hline
\end{tabular}

Red-only fluorescence to total red fluorescence ratio (\%) expressed as a "Mitophagy index" for each subject (Table 1) included in the experiments presented in Fig. 4A (mean of the 4 independent experiments). 